ARTICLE

\title{
Endothelial Unc5B controls blood-brain barrier integrity
}

Kevin Boyé (i) 1, Luiz Henrique Geraldo (1) 1,2, Jessica Furtado1, Laurence Pibouin-Fragner², Mathilde Poulet (iD) 1,2, Doyeun Kim³ ${ }^{3}$, Bryce Nelson ${ }^{4}$, Yunling $\mathrm{Xu}^{2}$, Laurent Jacob², Nawal Maissa², Dritan Agalliu (D) ${ }^{5}$, Lena Claesson-Welsh (iD ${ }^{6}$, Susan L. Ackerman ${ }^{7} \&$ Anne Eichmann ${ }^{1,2,8}{ }^{凶}$

Blood-brain barrier (BBB) integrity is critical for proper function of the central nervous system (CNS). Here, we show that the endothelial Unc5B receptor controls BBB integrity by maintaining $W n t / \beta$-catenin signaling. Inducible endothelial-specific deletion of Unc5B in adult mice leads to $B B B$ leak from brain capillaries that convert to a barrier-incompetent state with reduced Claudin- 5 and increased PLVAP expression. Loss of Unc5B decreases BBB Wnt/ $\beta$ catenin signaling, and $\beta$-catenin overexpression rescues Unc5B mutant BBB defects. Mechanistically, the Unc5B ligand Netrin-1 enhances Unc5B interaction with the Wnt coreceptor LRP6, induces its phosphorylation and activates $W n t / \beta$-catenin downstream signaling. Intravenous delivery of antibodies blocking Netrin-1 binding to Unc5B causes a transient BBB breakdown and disruption of Wnt signaling, followed by neurovascular barrier resealing. These data identify Netrin-1-Unc5B signaling as a ligand-receptor pathway that regulates $\mathrm{BBB}$ integrity, with implications for CNS diseases.

\footnotetext{
${ }^{1}$ Cardiovascular Research Center, Department of Internal Medicine, Yale University School of Medicine, New Haven, CT, USA. ${ }^{2}$ Université de Paris, INSERM, PARCC, F-75015 Paris, France. ${ }^{3}$ OliX pharmaceuticals, Suwon, Republic of Korea. ${ }^{4}$ Department of Pharmacology, Cancer Biology Institute, Yale University School of Medicine, New Haven, CT, USA. ${ }^{5}$ Departments of Neurology and Pathology and Cell Biology, Columbia University Irving Medical Center, New York, NY, USA. ${ }^{6}$ Department of Immunology, Genetics and Pathology, Uppsala University, Uppsala, Sweden. ${ }^{7}$ Division of Biological Sciences Section of Neurobiology and Department of Cellular and Molecular Medicine, University of California San Diego and Howard Hughes Medical Institute, La Jolla, CA, USA. ${ }^{8}$ Department of Molecular and Cellular Physiology, Yale University School of Medicine, New Haven, CT, USA. 凶email: anne.eichmann@yale.edu
} 
T he $\mathrm{BBB}$ protects the brain from toxins and pathogens and maintains homeostasis and proper function of the $\mathrm{CNS}^{1,2}$. To form the BBB, endothelial cells (ECs) lining brain blood vessels acquire a series of features that control the movement of ions, molecules and cells between the blood and the brain ${ }^{3}$. CNS ECs express specialized tight junctions that prevent paracellular exchange of small molecules ${ }^{4,5}$. They lack fenestrae and exhibit a low rate of transcytosis that limits transcellular exchange of large molecules ${ }^{6,7}$, and they express transporters that shuttle essential nutrients into and out of the brain ${ }^{8-10}$. ECs acquire BBB properties through their interactions with mural cells and glial cells in the CNS microenvironment and are modulated by neuronal activity $^{11-14}$.

The canonical Wnt/ $\beta$-catenin signaling pathway is a key regulator of BBB development and maintenance. CNS specific Wnt7a,7b and Norrin ligands produced by glial cells bind to multiprotein receptor complexes including Frizzled4 and LRP6 on brain ECs ${ }^{15-21}$. Receptor activation causes $\beta$-catenin stabilization and nuclear translocation to induce activation of TCF and LEF1 transcription factors, which control expression of a BBBspecific gene expression repertoire by inducing tight junction, solute transporter and efflux transporter expression, and by repressing expression of the permeability protein PLVAP that forms the diaphragm in EC fenestrae, caveolae and transcytotic vesicles ${ }^{15,22-24}$. Whether $\mathrm{Wnt} / \beta$-catenin signaling could be modulated to open the BBB "on-demand" or to restore its integrity when damaged, is unknown.

Unc5 was initially discovered in a C. elegans screen for motor dysfunctions, and controls axon guidance in all species examined 25,26 . Among the four vertebrate Unc5 family members, only Unc5B is expressed in ECs in mice and humans ${ }^{27}$. Unc5B binds Netrin-1 $1^{26,28}$, Robo4 ${ }^{29,30}$ and Flrt2 $2^{31,32}$ via its extracellular domain (ECD). Unc5B signaling is mediated by its intracellular domain (ICD), which encompasses a membrane-proximal ZU5 domain (named for its homology to ZO1), followed by a UPA domain (named for its conservation in Unc5B, PIDD and Ankyrin $^{33}$ ) and a death domain (DD) that mediates apoptosis in the absence of ligand ${ }^{33,34}$. These domains form a supramodule in which ZU5 binds to both UPA and DD suppressing Unc5B biological function, while ligand binding to Unc $5 \mathrm{~B}$ triggers a conformational change such that ZU5 loses its interaction with DD and exposes the UPA domain to activate Unc5B signaling ${ }^{33}$. Global homozygous Unc5B knockout in mice is embryonically lethal due to placental vascular defects 27,31 , demonstrating that Unc5B has important functions in vascular development. To address biological roles of this receptor during postnatal and adult life, we generated mice with temporally inducible, endothelial-specific Unc5B deletions.

Here, we identify the endothelial transmembrane Unc5B receptor and its ligand Netrin-1 as regulators of the BBB integrity. Inducible endothelial-specific Unc5B deletions in adult mice leads to reduced $\mathrm{Wnt} / \beta$-catenin signaling and BBB leak for tracers up to $40 \mathrm{kDa}$. Unc5B and $\beta$-catenin genetically interact in ECs to maintain BBB integrity and overexpressing an activated form of $\beta$-catenin rescues BBB defects induced by loss of Unc5B. We show that intravenous delivery of monoclonal antibodies blocking Netrin-1 binding to Unc5B induce transient $\mathrm{BBB}$ opening to bioactive molecules, which could be useful for drug delivery in various neurological diseases.

\section{Results}

Endothelial Unc5B controls BBB development and maintenance. We generated $U n c 5 B^{f l / f l}$ mice (Supplementary Fig. 1a) that were intercrossed with $C d h 5 C r e^{E R T 2}$ mice (hereafter Unc5BiECko), which produces EC-specific deletion ${ }^{35}$. Gene deletion was induced by Tamoxifen (TAM) injection in neonates between postnatal day (P)0-P2, and qPCR revealed efficient Unc $5 B$ deletion (Supplementary Fig. 1b, c). Interestingly, neonatal TAM injection-induced seizures and lethality of Unc5BiECko mice around P12 (Supplementary Fig. 1d, Supplementary movie), indicating a possible $\mathrm{BBB}$ failure ${ }^{2}$. Intraperitoneal injection of a fluorescent tracer cadaverine (MW 950 Da) into P5 mice and analysis of tracer leak $2 \mathrm{~h}$ later revealed widespread tracer extravasation into the brain of P5 Unc5BiECko mice, confirming that Unc5B deletion impaired BBB development (Supplementary Fig. 1e).

To determine if Unc5B also controlled BBB integrity in adults, we induced gene deletion in two months old mice and probed BBB integrity 7 days later by intravenous (i.v.) cadaverine injection (Fig. 1a). Mice were sacrificed 30 min after dye injection and brain sections were immunolabeled with an antibody against podocalyxin, which labels the luminal EC membrane ${ }^{36}$. Cadaverine remained inside the vasculature of TAM-injected Crenegative littermate controls but leaked into the Unc5BiECko brain (Fig. 1b). Quantification of cadaverine extravasation (see methods) revealed significantly increased leak across the BBB in Unc5BiECko brains when compared to controls, while the vascular permeability to cadaverine in other Unc5BiECko organs was similar to controls (Fig. 1C), indicating that Unc5B has a CNS-selective BBB-protective function in adult mice.

Staining of adult brain sections with a commercial antibody recognizing Unc5B showed labeling of endothelium in various brain regions and revealed that adult Unc5B deletion had no effect on vascular density over the period observed (Fig. 1d, e) Widespread but non-uniform cadaverine leakage was observed in several brain regions of Unc5BiECko mice, including the retrosplenial and piriform cortex, hippocampus, hypothalamus, thalamus, striatum and cerebellum, while other cortical areas such as the posterior parietal association areas and the primary somatosensory cortex displayed less cadaverine leak (Fig. 1f). Unc5B expression in cortical ECs of wildtype mice was similar between areas that displayed more or less cadaverine leak in Unc5BiECko brains (Fig. 1g), hence Unc5B expression alone was not sufficient to predict severity of BBB breakdown.

Unc5B expression was also detected in some $\mathrm{CD} 13^{+}$pericytes (Fig. 1h). Because pericytes contribute to BBB integrity ${ }^{11,12}$, we determined whether pericyte-derived Unc5B affected the BBB by crossing the Unc5B $B^{f l f l}$ mice with $P d g f r \beta C r e^{E R T 2}$ mice $^{37}$ (Unc5BiPCko), to delete Unc5B in mural cells. Neither TAMtreated Cre-negative littermate controls, nor Unc5BiPCko mice showed cadaverine leakage across the BBB (Fig. 1i, j). Hence, endothelial, but not pericyte, Unc5B controls adult BBB integrity.

Endothelial Unc5B controls Claudin-5 and PLVAP expression. To determine the cause of the $\mathrm{BBB}$ defect, we examined the expression levels of proteins relevant for BBB function in ECs, pericytes and astrocytes by western blot and immunolabeling ${ }^{5,11-13,38}$. Compared to TAM-treated Cre-negative littermate controls, Unc5BiECko brains had similar expression of EC BBB regulators such as Caveolin-1, ZO1, and Occludin (Fig. $2 \mathrm{a}-\mathrm{c})$. In addition, vessel coverage by PDGFR $\beta+$ pericytes, and GFAP and Aquaporin-4 expression in astrocytes and astrocyte endfeet were also similar between genotypes (Fig. 2a-e). By contrast, Unc5BiECko mice showed significantly reduced expression of Claudin-5, along with increased expression of PLVAP in Western blot analysis of brain lysates (Fig. 2f, g). Immunolabeling of adult brain sections showed decreased Claudin-5 and increased PLVAP expression in areas with cadaverine leakage in Unc5BiECko brains (Fig. $2 \mathrm{~h}$ ), suggesting that BBB leakage may be due to changes in expression of Claudin-5 
a

b
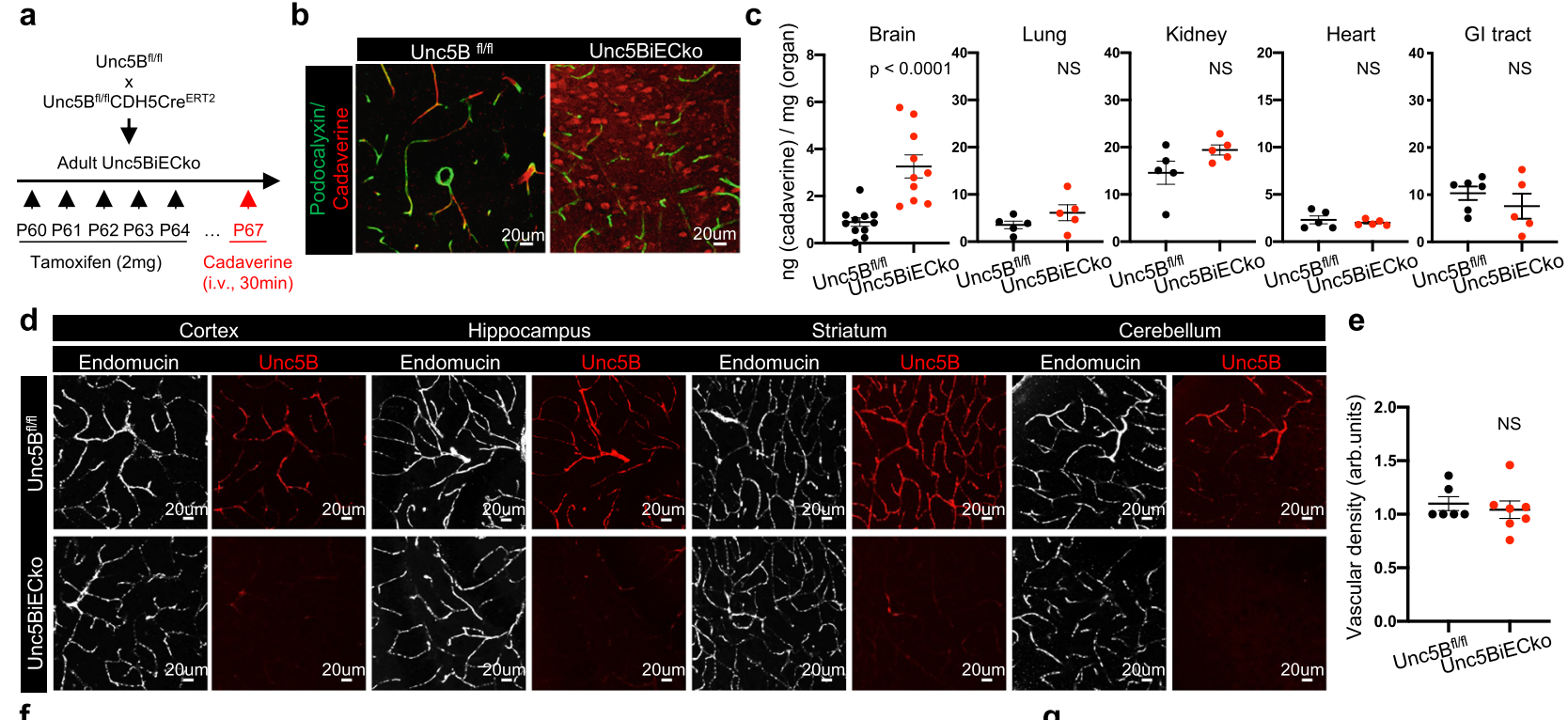

e
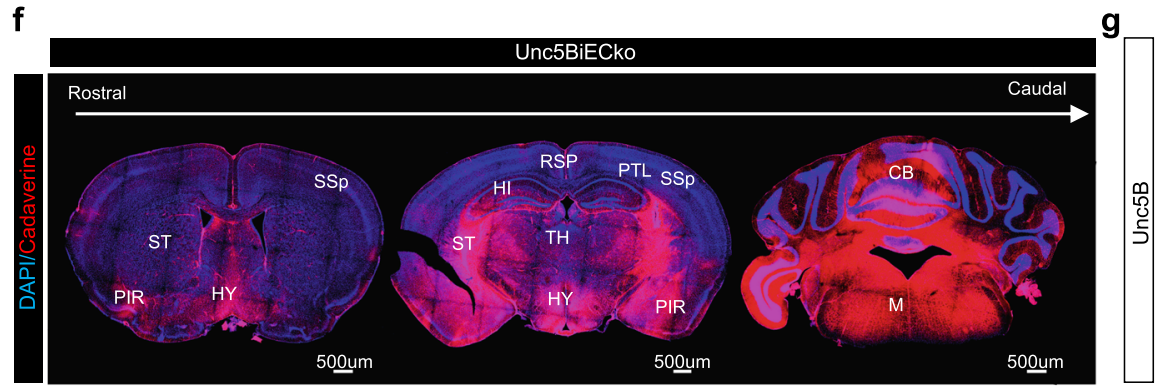

h

i

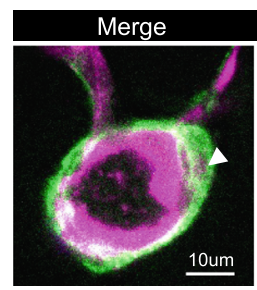
Unc5B
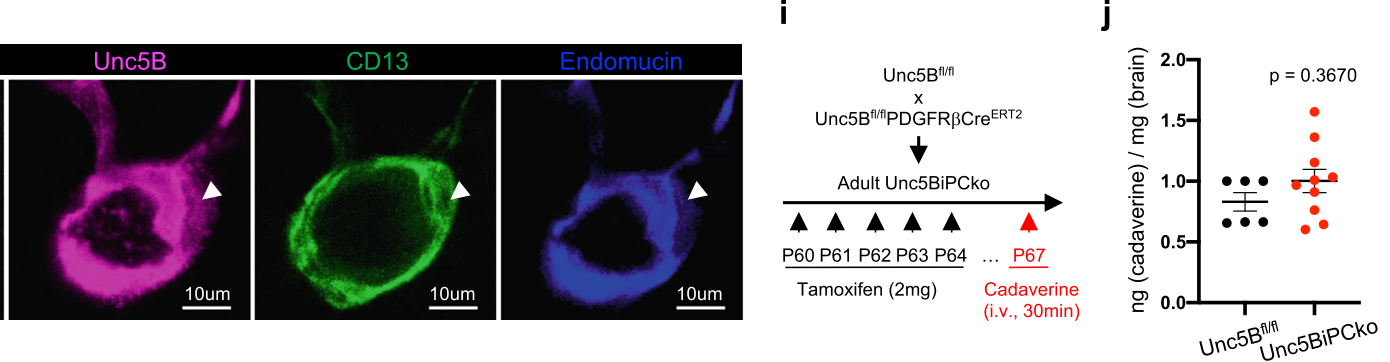

Fig. 1 Endothelial Unc5B controls BBB integrity. a Unc5B gene deletion strategy using tamoxifen injection in adult mice. $\mathbf{b}$ Immunofluorescence staining with the indicated antibodies and confocal imaging of adult brain sections at P67, 30 min after i.v cadaverine injection and reproduced on $n=4$ Unc5B $\mathrm{fl}^{\mathrm{l} f l}$ and $n=5$ Unc5BiECko brains. c Quantification of dye content in brains and peripheral organs at P67, 30 min after i.v. cadaverine injection, $n=11$ Unc5 $B^{f / f l}$ and $n=10$ Unc5BiECko brains (exact $p$-value $=0.0000397$ ); $n=5$ Unc5Bfl/fl and $n=10$ Unc5BiECko lungs, kidneys and hearts; $n=6$ Unc5Bfl/fl and $n=5$ Unc5BiECko Gl tracts. Each dot represents one mouse. $\mathbf{d}$ Immunofluorescence staining with the indicated antibodies and confocal imaging of adult brain sections, reproduced on $n=4 U n c 5 B^{f l / f l}$ and $n=4$ Unc5BiECko brains. e Quantification of endomucin+ vascular density. Each dot represents the mean of several images, $n=6$ Unc5B $\mathrm{Bl}^{\mathrm{fl} I}$ and $n=7$ Unc5BiECko brains. One control mouse value was set as 1 . f Tile-scan confocal imaging of adult Unc5BiECko brain sections at P67, 30 min after i.v cadaverine injection and reproduced on $n=3$ Unc5B $B^{f / f l}$ and $n=3$ Unc5BiECko brains. $\mathbf{g}$ Immunofluorescence staining of Unc5B and tile-scan confocal imaging of brain sections, reproduced on $n=4$ mice. Boxes show higher magnifications of cortical areas. $\mathbf{h}$ Immunofluorescence staining with the indicated antibodies and confocal imaging of adult brain sections, reproduced on $n=4$ Unc5Bfl/fl and $n=4$ Unc5BiECko brains. Arrowhead: Unc5B+/CD13+ pericyte. $\mathbf{i}, \mathbf{j}$ Unc5B $\mathrm{Bl}^{\mathrm{f} / \mathrm{fl}}$ was crossed with PDGFR $\beta C$ re ${ }^{E R T 2}$ and BBB permeability was assessed at P67, 30 min after i.v. cadaverine injection, $n=6$ Unc5B fl/fl and $n=10$ Unc5BiPCko brains. Each dot represents one mouse. All data are shown as mean \pm SEM. NS non-significant, RSP Retrosplenial cortex, PTL Posterior parietal association areas, SSp Primary somatosensory cortex, PIR Piriform cortex, HI Hippocampus, HY Hypothalamus, TH Thalamus, ST Striatum, CB Cerebellum, M Medulla. Two-sided Mann-Whitney $U$ test was performed for statistical analysis. Source data are provided as a Source Data file.

and PLVAP. Conversion of ECs to a Claudin-5-negative, PLVAPpositive state occurred in Unc5BiECko capillaries but not in larger vessels ( $>10 \mathrm{um}$ in diameter, Fig. $2 \mathrm{~h}$, Supplementary Fig. 2a), suggesting that BBB leakage in Unc5BiECko brains originates from capillaries. To validate Unc5B deletion effects on Claudin-5 and PLVAP in an independent knockout mouse strain, we tested global Unc5B KO embryos at E12.5 ${ }^{27}$, which confirmed decreased CLDN5 mRNA and protein levels as well as increased PLVAP protein expression in homozygous mutant brain ECs when compared to wildtype control littermate brains (Supplementary Fig. 2b-e).

Unc5B regulates $\mathrm{Wnt} / \boldsymbol{\beta}$-catenin signaling. Because Claudin-5 and PLVAP are two known targets of $\mathrm{CNS} W n t / \beta$-catenin signaling $22-24$, we determined if Unc5B affected Wnt signaling at the BBB. qPCR on adult brain lysates revealed decreased mRNA 
a

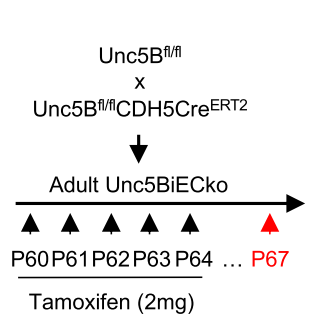

b

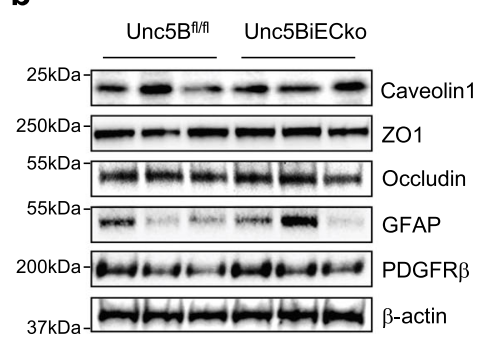

C

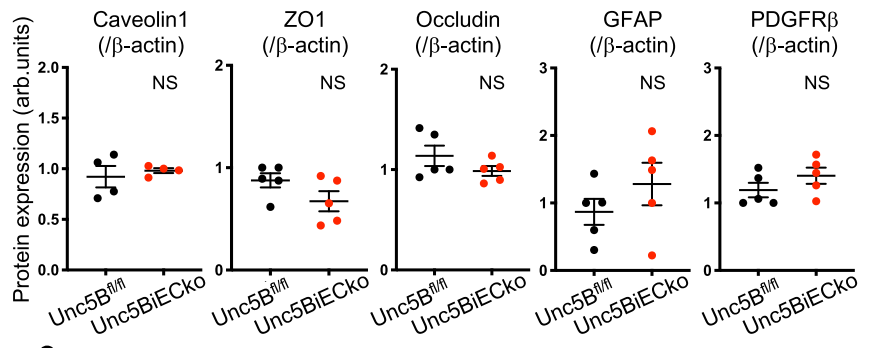

e

d
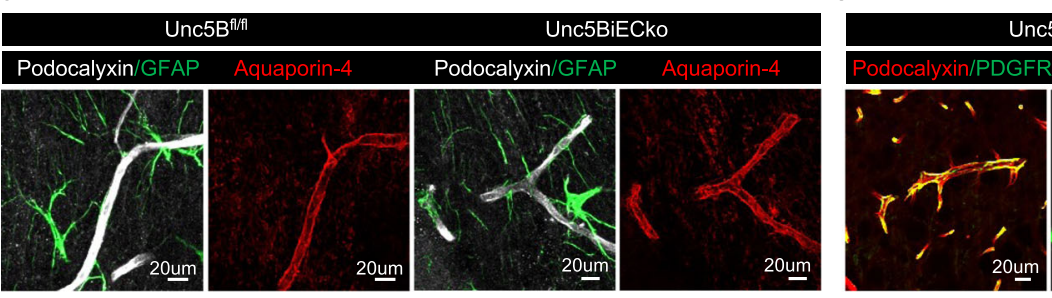

Unc5B $\mathrm{B}^{\mathrm{f} / \mathrm{fl}}$

Unc5BiECko

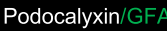

Aquaporin-4
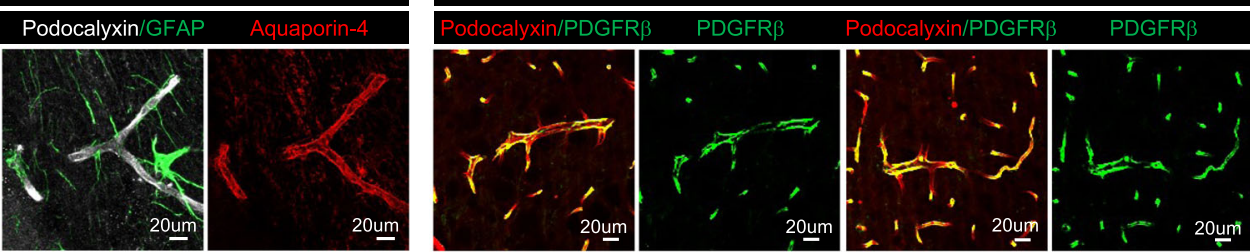

$\mathbf{f}$

h

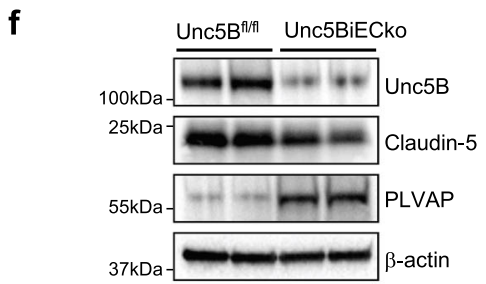

g
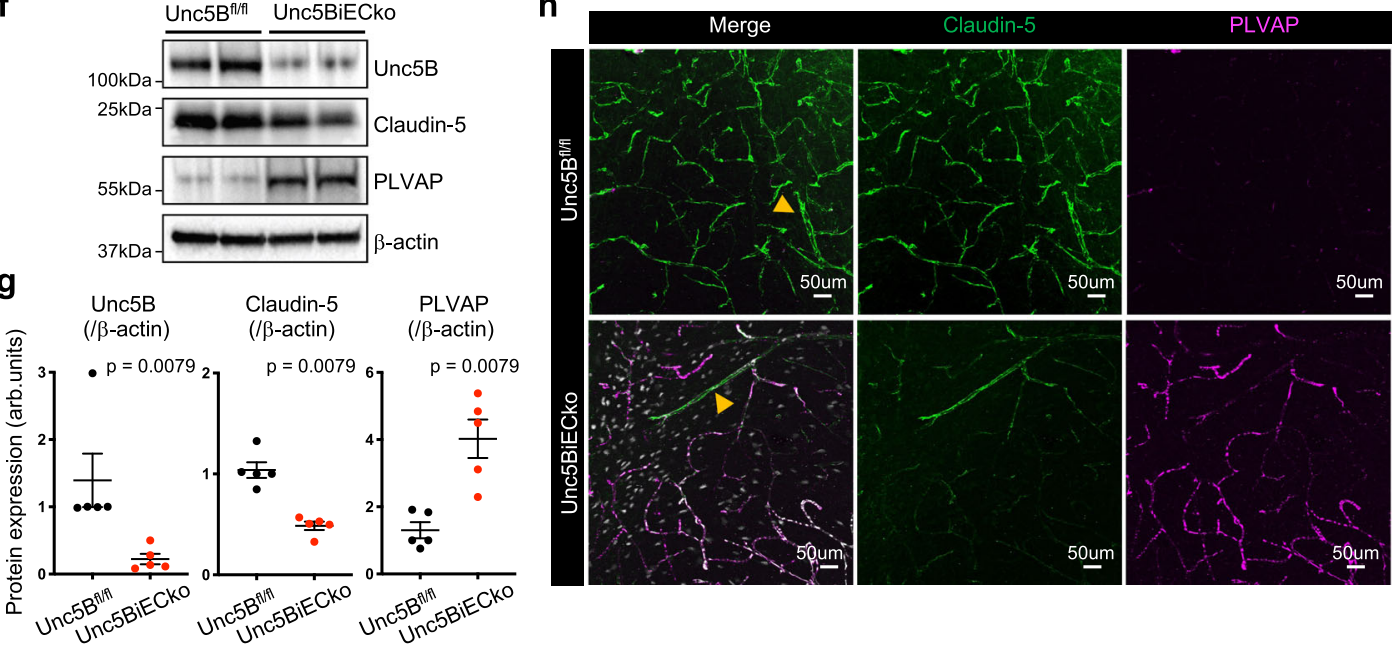

Cadaverine

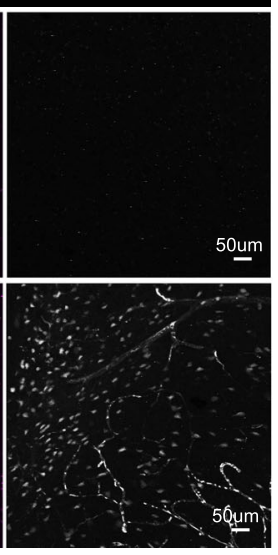

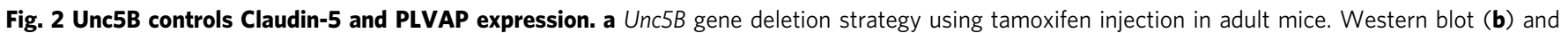

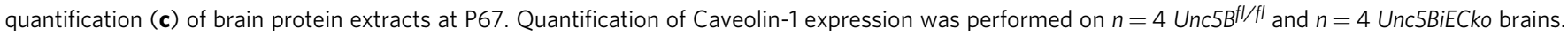
Quantification of ZO1, Occludin, GFAP and PDGFR $\beta$ expression was performed on $n=5$ Unc5Bfl/fl and $n=5$ Unc5BiECko brains. Each dot represents one mouse. One control mouse was set as 1. d, e Immunofluorescence staining with the indicated markers and confocal imaging of brain sections, reproduced on $n=4$ Unc5B fl/fl and $n=4$ Unc5BiECko brains. Western blot (f) and quantification ( $\mathbf{g}$ ) of P67 brain protein extracts, $n=5$ Unc5Bfl/fl and $n=5$ Unc5BiECko brains. Each dot represents one mouse. One control mouse value was set as 1. h Immunofluorescence staining with the indicated antibodies and confocal imaging of P67 piriform cortex 30 min after i.v cadaverine injection, reproduced on $n=4$ Unc5B fl/fl and $n=4$ Unc5BiECko brains. Arrowheads: larger

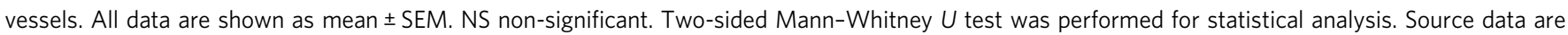
provided as a Source Data file.

levels of $L E F 1$ and CLDN5 as well as increased PLVAP mRNA levels in Unc5BiECko brains compared to Cre-negative littermate controls (Fig. 3a). Next, we crossed Unc5BiECko mice with TCF/ LEF:H2B-GFP mice $^{39}$ which express a GFP reporter of $\beta$-catenin transcriptional activity. Compared to TAM-treated Cre-negative controls, Unc5BiECko;TCF/LEF:H2B-GFP brains revealed decreased GFP expression in ECs nuclei labeled with the endothelial transcription factor $\mathrm{ERG}^{40}$, attesting to decreased endothelial $\beta$-catenin transcriptional activity upon loss of Unc5B function (Supplementary Fig. 3a-c). Western-blot analysis on brain lysates revealed a significant decrease of $\beta$-catenin and LEF1 protein in Unc5BiECko brains compared to Cre-negative littermate controls (Fig. 3b, c). Moreover, phosphorylation of LRP6 at S1490, a hallmark of $\mathrm{Wnt} / \beta$-catenin pathway activation ${ }^{41}$, was dramatically downregulated upon $U n c 5 B$ gene deletion (Fig. $3 \mathrm{~b}$, c). This phosphorylation provides a docking site for the adapter protein Axin1, resulting in inhibition of the $\beta$-catenin destruction complex and thereby promoting $\beta$-catenin nuclear translocation and activation ${ }^{41,42}$. Immunostaining confirmed decreased LEF1 expression in brain ECs of Unc5BiECko mice (Fig. 3d, e).

Immunoprecipitation of Unc5B from primary microvascular mouse brain ECs pulled down LRP6, pLRP6, Frizzled4 and the Wnt co-receptor GPR124 (Fig. 3f), demonstrating a physical interaction between Unc5B and Wnt receptors. To determine which Unc5B domain mediated this interaction, we infected Unc5B siRNA-treated human ECs with GFP-tagged siRNA resistant rat adenoviral constructs encoding Unc5B full-length (FL) or a cytoplasmic domain deletion $(\Delta \mathrm{CD})$ (Fig. 3g). LRP6 coIP was rescued by Unc5B FL but not by $\triangle \mathrm{CD}$, identifying the Unc5B cytoplasmic domain as the main LRP6 interacting domain. Additional cytoplasmic domain deletions revealed that the Unc5B death domain was dispensable for LRP6 interaction, whereas deletion of the UPA domain abolished LRP6 co-IP. A construct encoding only the cytoplasmic UPA domain was 
a

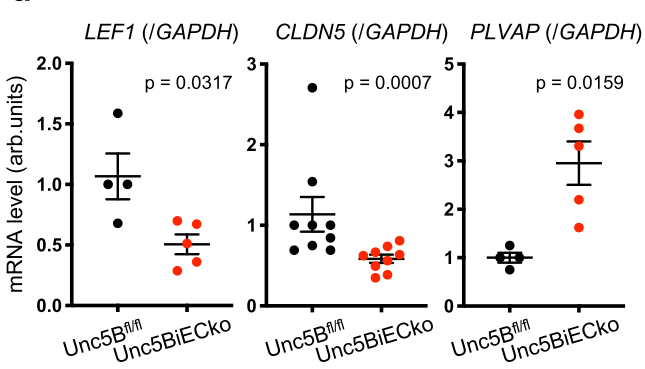

b

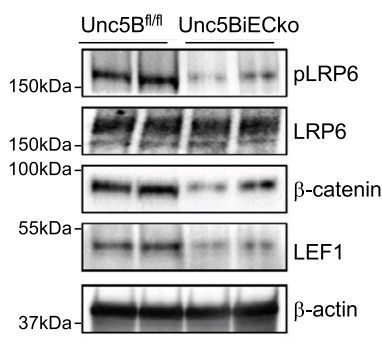

C

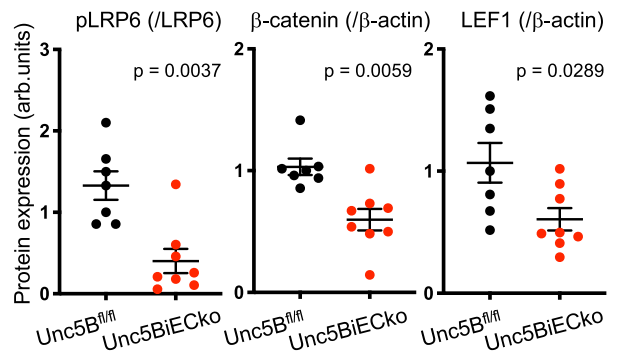

d

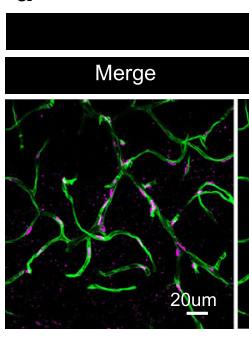

f

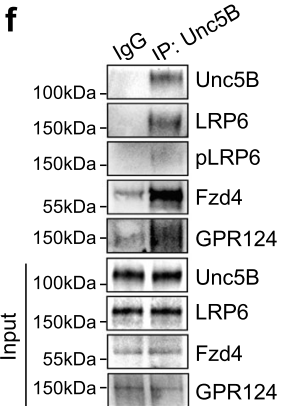

j

Unc5B $B^{\text {IlwT }}$ CDH5Cre ${ }^{\text {ERT2 }}$

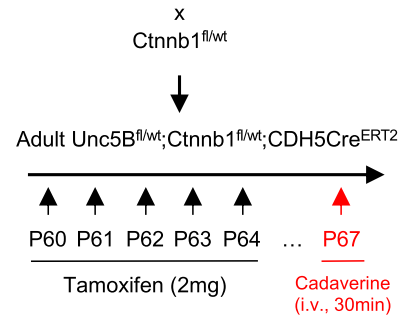

m

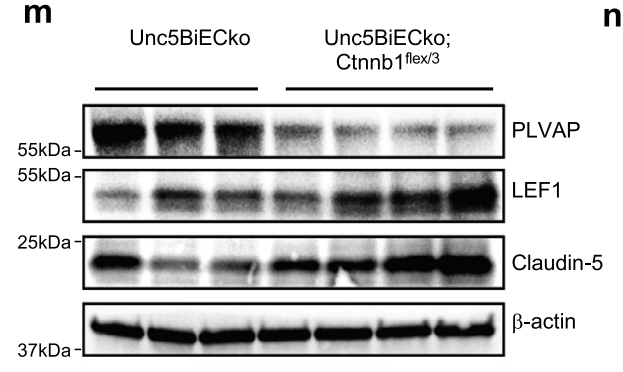

g 20um

20um

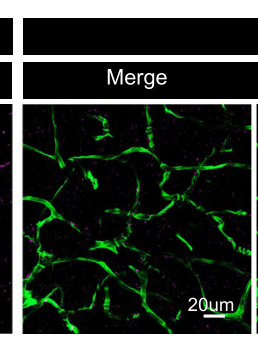

h

\section{e}

LEF1 (/Podocalyxin)
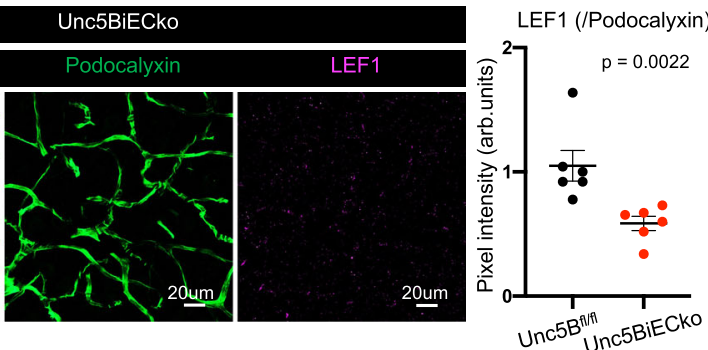

i
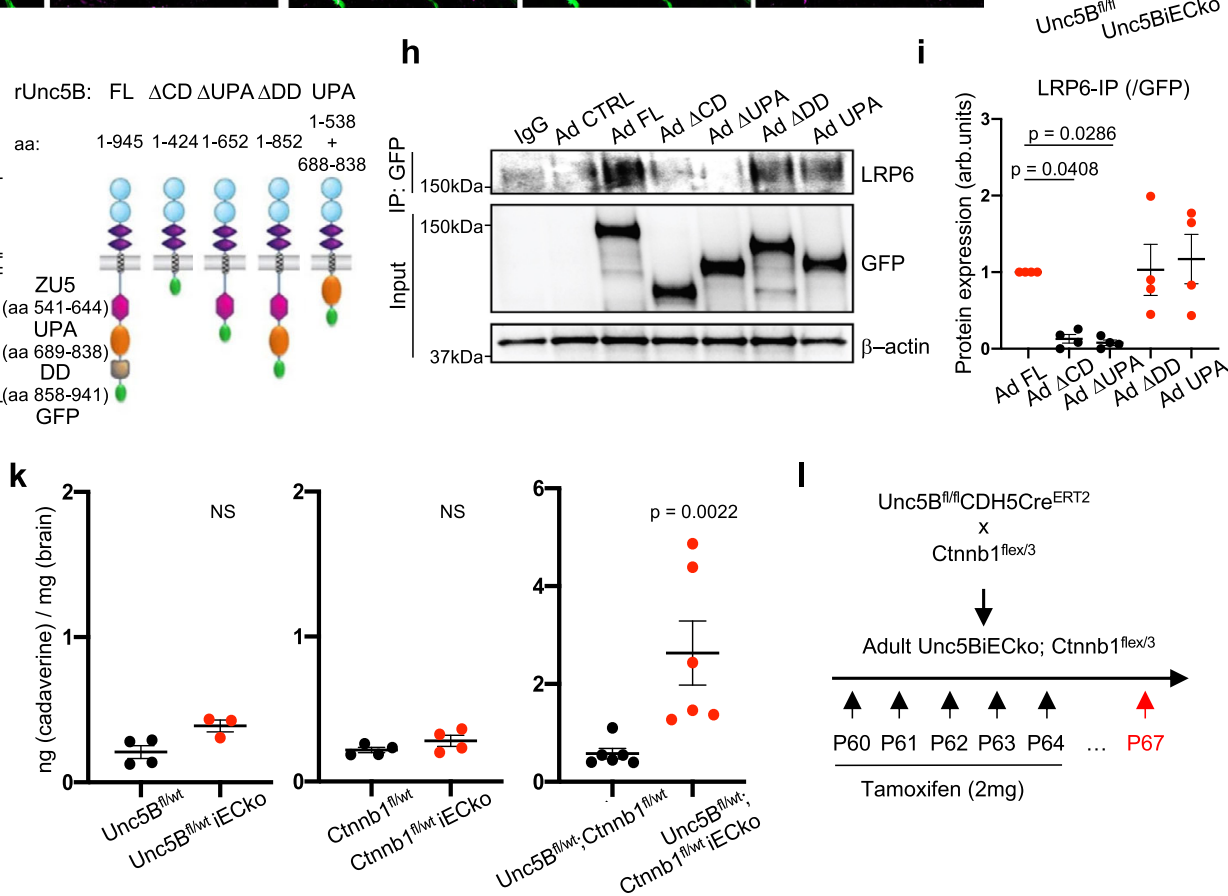

I

n $\quad \operatorname{PLVAP}(/ \beta$-actin $)$

LEF1 (/ $\beta$-actin) Claudin-5 (/ $\beta$-actin)
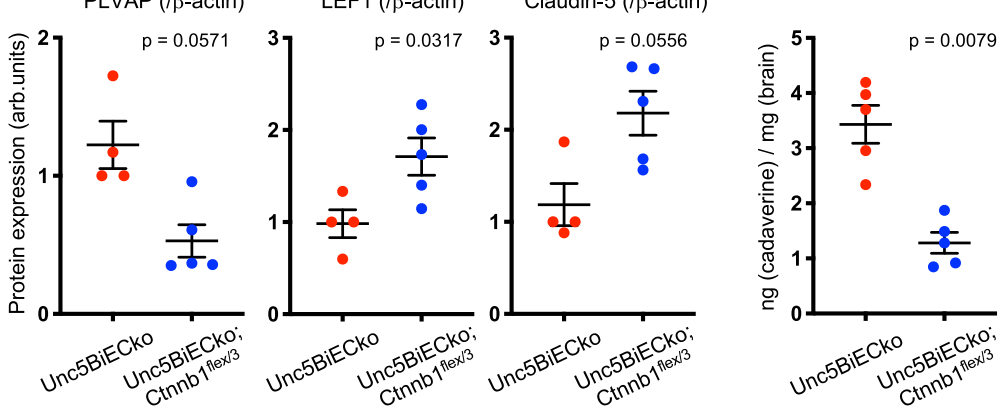

sufficient to rescue LRP6 co-IP (Fig. 3h, i), indicating that Unc5B interacts with LRP6 via its UPA domain.

To test genetic interaction of Unc5B with $\mathrm{Wnt} / \beta$-catenin signaling, we generated a TAM-inducible endothelial-specific $\beta$ catenin deletion by crossing Ctnnb1 $1^{f l f l}$ mice $^{43}$ with $C D H 5 C r e^{E R T 2}$ mice (Fig. 3j, Supp. Figure 3d-f). Heterozygous $U n c 5 B^{f l w t}$ $C D H 5 C r e^{E R T 2}$ or Ctnnb1 fl/wt $C D H 5 C r e^{E R T 2}$ mice displayed no
$\mathrm{BBB}$ cadaverine leakage, but $\mathrm{BBB}$ cadaverine leakage increased in double heterozygous Unc5B ${ }^{f l / w t}$;Ctnnb $1^{f l w t}-C D H 5 C r e^{E R T 2}$ brains compared to TAM-treated Cre-negative controls (Fig. 3j, k), demonstrating that $U n c 5 B$ and $\beta$-catenin genetically interact in ECs to maintain BBB integrity.

Next, we crossed Unc5BiECko with mice overexpressing a TAM-inducible activated form of $\beta$-catenin (Ctnnb1 $1^{\text {flex } / 3}$ mice $^{44}$ ), 
Fig. 3 Unc5B regulates BBB Wnt/ק-catenin signaling. a qPCR analysis of P67 brain mRNA extracts, $n=4$ Unc5B fl/fl and $n=5$ Unc5BiECko brains for quantification of LEF1 and PLVAP mRNA levels, $n=9$ Unc5B fl/fl and $n=9$ Unc5BiECko brains for quantification of CLDN5 mRNA levels. Each dot represents one mouse. One control mouse was set as 1 . Western blot (b) and quantification (c) of Wnt/ $\beta$-catenin signaling components in brain protein extracts,

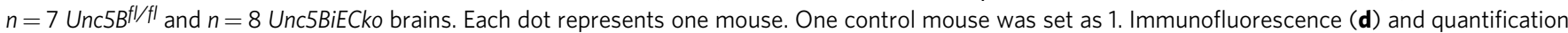
(e) of LEF1 staining on adult brain sections. Each dot is the mean of several images, $n=6$ Unc5 $\mathrm{B}^{\mathrm{fl} / \mathrm{fl}}$ and $n=6$ Unc5BiECko brains. One control mouse was set as 1. $\mathbf{f}$ CTRL IgG and Unc5B immunoprecipitation on cultured brain endothelial cells, reproduced on $n=3$ independent experiment. $\mathbf{g}$ Schematic of Unc5B adenoviral constructs. CTRL IgG or GFP immunoprecipitation in Unc5B siRNA knockdown ECs infected with siRNA resistant Unc5B adenovirus (h), and quantification of LRP6 pulldown (i), $n=4$ independent experiment. Each dot represents one independent experiment. $\mathbf{j} U n c 5 B$ and $C$ tnnb1 gene deletion strategy using tamoxifen injection in adult mice. $\mathbf{k}$ Quantification of cadaverine content in P67 brains, 30 min after i.v. cadaverine injection, $n=4$ Unc5 $B^{f / / w t}$,

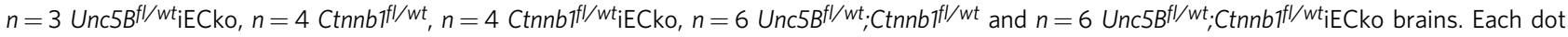

represents one mouse. I Unc5B gene deletion and $C$ tnnb flex/3 gene overexpression strategy using tamoxifen injection. Western blot ( $\mathbf{m}$ ) and quantification (n) of P67 brain protein extracts, $n=4$ Unc5BiECko and $n=5$ Unc5BiECko;Ctnnb7lex/3 brains. Each dot represents one mouse. One control mouse was set as 1. 0 Quantification of P67 brain cadaverine content, 30 min after i.v cadaverine injection, $n=5$ Unc5BiECko and $n=5$ Unc5BiECko;Ctnnbflex/3 brains. Each dot represents one mouse. All data are shown as mean \pm SEM. NS non-significant. Two-sided Mann-Whitney $U$ test was performed for statistical analysis between two groups. ANOVA followed by Bonferroni's multiple comparisons test was performed for statistical analysis between multiple groups. Source data are provided as a Source Data file.

thereby enhancing endothelial $\mathrm{Wnt} / \beta$-catenin signaling $22,24,45$ (Fig. 31, Supplementary Fig. 3g-i). The resulting offspring (Unc5BiECko; Ctnnb1 flex/3) displayed decreased PLVAP protein expression, along with increased LEF1 and Claudin-5 protein expression compared to Unc5BiECko mice (Fig. $3 \mathrm{~m}, \mathrm{n}$ ). Cadaverine injection into Unc5BiECko;Ctnnb1 flex $/ 3$ mice showed that $\mathrm{BBB}$ leakage was reduced by $\beta$-catenin overexpression in Unc5BiECko mice (Fig. 3o). Western-blot on brain lysates from

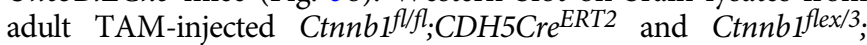
CDH5Cre ${ }^{E R T 2}$ mice showed that loss or gain of endothelial $\beta$ catenin did not significantly change Unc5B or Netrin-1 expression (Supplementary Fig. 3d-i), suggesting that Unc5B acted upstream of Wnt signaling.

Claudin-5 but not Vegfr2 is required for Unc5B BBB regulation. To test if reduced Claudin-5 expression in Unc5BiECko brains was functionally involved in the Unc5BiECko BBB defect, we crossed Unc5BiECko mice with eGFP::Claudin-5 mice that express 2-fold higher Claudin-5 levels compared to wildtype littermates and thereby display enhanced CNS EC paracellular barrier properties ${ }^{46}$. BBB leakage of cadaverine into the brains of Unc5BiECko; eGFP::Claudin-5 mice was reduced compared to Unc5BiECko mice (Fig. 4a, b), demonstrating that increasing Claudin-5 levels rescued leakage of small MW tracers in Unc5B mutant brain ECs.

We considered other signaling pathways that could contribute to BBB leakage in Unc5BiECko mice. Unc5B inhibits Vegfr2mediated permeability signaling in ECs in vitro by reducing phosphorylation of the Y949 residue ${ }^{30}$. Y949 phosphorylation is known to trigger disassembly of adherens junctions by activating VE-cadherin phosphorylation, which then downregulates Claudin-529,30,47. Therefore, increased brain Vegfr2-Y949 permeability signaling in the absence of Unc5B could contribute to BBB opening. Western blotting of brain lysates revealed increased Vegfr2-Y949 phosphorylation in Unc5BiECko compared to Crenegative littermate controls, while Vegfr2-Y1173 phosphorylation, which is critical for VEGF-induced proliferation, was unaffected (Fig. 4c, d). To test Vegfr2-Y949 function, we crossed Unc5BiECko mice with Vegfr2-Y949F mutant mice, which carry an inactivating substitution of tyrosine to phenylalanine and are resistant to VEGF-induced permeability ${ }^{48}$. Injection of fluorescent cadaverine revealed increased dye leakage into the brain of Unc5BiECko; Y949F mice compared to Cre-negative littermate controls (Fig. 4e, f), demonstrating that Vegfr2-Y949F failed to rescue $\mathrm{BBB}$ integrity in Unc5B mutant mice. Moreover, similar VE-cadherin expression and junctional coverage were observed in CTRL and Unc5BiECko brains, further attesting that Unc5B regulation of $\mathrm{BBB}$ integrity is independent of the Vegfr2 VEcadherin pathways (Fig. $4 \mathrm{~g}-\mathrm{j}$ ).

Netrin-1 controls BBB integrity. We determined whether Unc5B ligands Netrin-1 and Robo4 regulated BBB integrity and $W n t / \beta$ catenin pathway activation in CNS ECs. Since Netrin-1 mRNA is produced by several cell types in the adult brain ${ }^{49}$, we generated temporally inducible Netrin-1 global KO mice by crossing Ntn $1^{f l f l}$ mice $^{50}$ with RosaCre $e^{E R T 2}$ mice (hereafter Ntn1iko), to induce ubiquitous gene deletion upon TAM injection. Compared to TAM-treated Cre-negative littermate controls, i.v. injection of cadaverine in adult Ntn1iko mice revealed increased cadaverine leakage across the BBB (Fig. 5a), while Robo4 $\mathrm{KO}$ mice ${ }^{29}$ did not exhibit any BBB leakage (Fig. 5b). Further analysis of adult Ntn1iko mouse brain lysates revealed efficient Ntn1 gene deletion along with decreased pLRP6, Claudin-5 and LEF1 protein expression, while PLVAP expression was increased (Fig. $5 c$, d). Moreover, treating serum-starved mouse primary brain ECs with Netrin-1 increased LRP6 phosphorylation with a peak at $30 \mathrm{~min}$ to $8 \mathrm{~h}$ after stimulation (Fig. 5e, f). This effect was abolished by Unc5B siRNA treatment (Fig. 5e, f). Unc5B immunoprecipitation from mouse brain lysates revealed reduced LRP6 co-IP in the Ntnliko mice when compared to controls (Fig. 5g, h), suggesting that Netrin-1 binding to Unc5B regulated LRP6 phosphorylation and Wnt/ $\beta$-catenin activation in CNS ECs. We reasoned that Netrin-1 could modulate LRP6 phosphorylation via FAK, a kinase that regulates $\beta$-catenin in pluripotent embryonic stem cells ${ }^{51}$. Netrin-1-treated mouse brain ECs showed increased FAK phosphorylation from 1 to $8 \mathrm{~h}$ after stimulation (Fig. 5i, j). Nevertheless, cells treated with a FAK inhibitor (FAKi) that effectively abolished FAK phosphorylation could still induce LRP6 phosphorylation upon Netrin-1 stimulation (Fig. 5k, l) demonstrating that Netrin-1 regulates LRP6 activation in brain ECs independently of FAK.

Netrin-1 binding to Unc5B mediates BBB integrity. To specifically interrogate whether blocking Netrin-1-Unc5B interactions disrupted the $\mathrm{BBB}$ in vivo, we used monoclonal antibodies (mAbs) that we had previously generated against the Unc5B IgGlike domains ${ }^{29}$. Anti-Unc5B-1 recognizes human but not mouse Unc5B, while anti-Unc5B-2 recognizes both human and mouse Unc5B and internalizes Unc5B ${ }^{29}$. Anti-Unc5B-2 treatment induced Unc5B internalization in brain ECs in vitro (Supplementary Fig. 4a) and i.v. injection of anti-Unc5B-2 for $1 \mathrm{~h}$ at $10 \mathrm{mg} / \mathrm{kg}$ in mice reduced brain Unc5B expression compared to anti-Unc5B-1 CTRL Ab-treated animals (Supplementary Fig. 4b, 
a

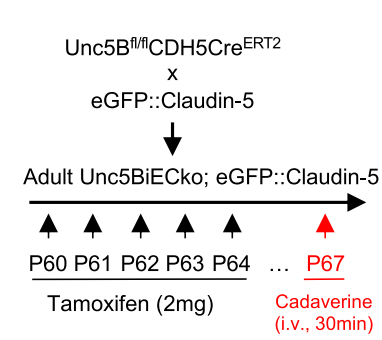

e

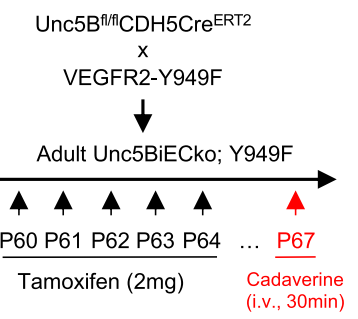

i

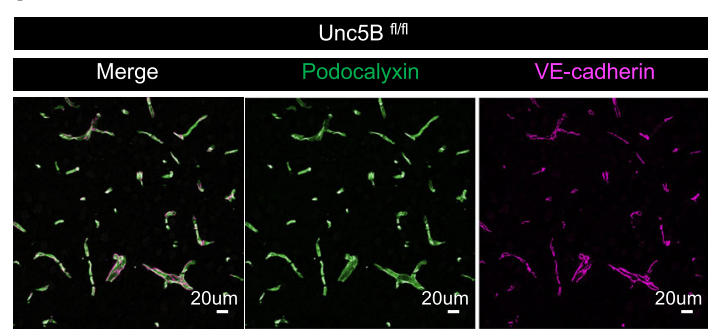

b

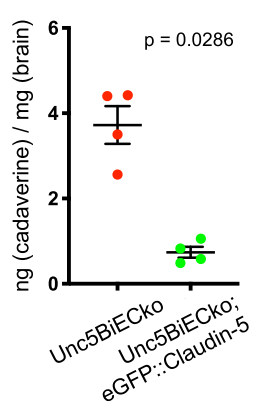

f

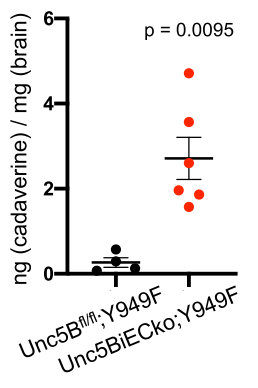

\section{$+$}

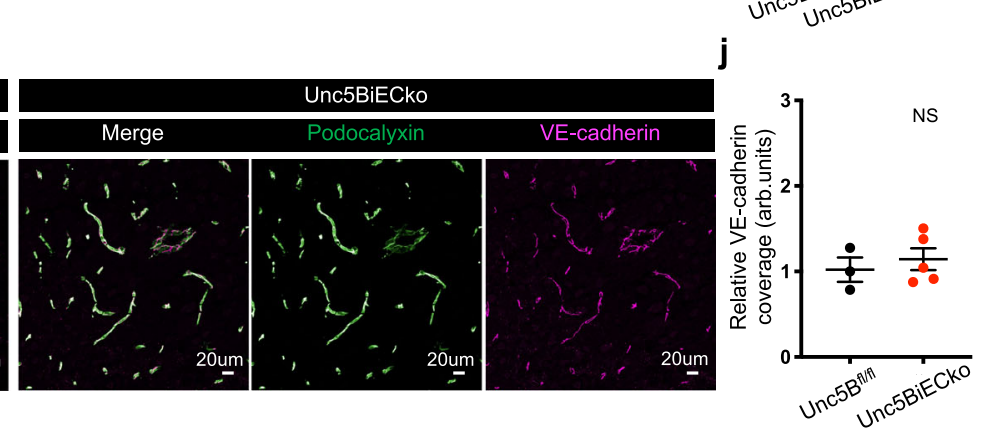

C

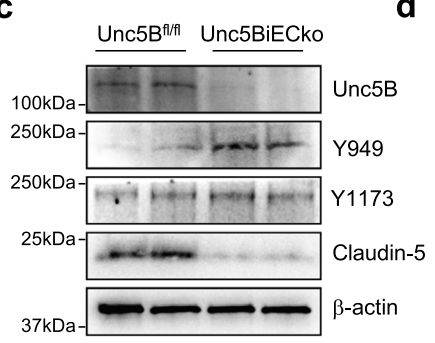

g

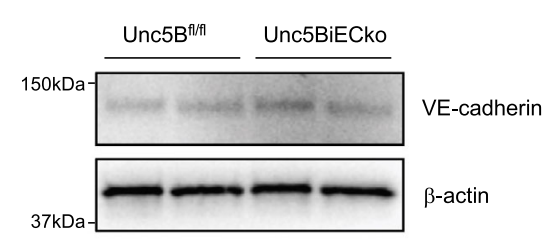

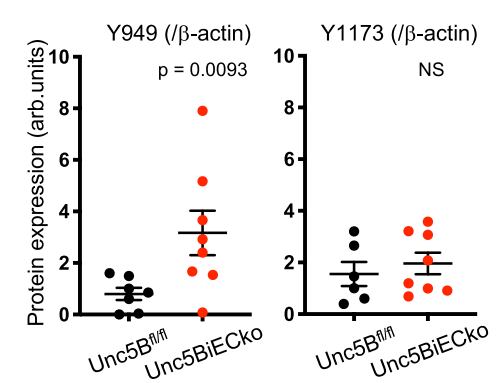

$\mathbf{h}$
VE-cadherin ( $\beta$-actin)

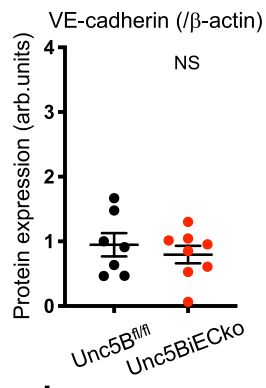

Fig. 4 Claudin-5 but not VEGFR2 is involved in Unc5B BBB regulation. a Unc5B deletion and eGFP::Claudin-5 gene overexpression strategy using tamoxifen injection. b Quantification of P67 brain cadaverine content, 30 min after i.v cadaverine injection, $n=4$ Unc5BiECko and $n=4$

Unc5BiECko; eGFP::Claudin-5 brains. Each dot represents one mouse. Western blot (c) and quantification (d) of P67 brain protein extracts, $n=7$ Unc5B fl/fl and $n=8$ Unc5BiECko brains. Each dot represents one mouse. One control mouse was set as 1. e Unc5B and VEGFR2-Y949F gene recombination strategy using tamoxifen injection. $\mathbf{f}$ Quantification of P67 brain cadaverine content, $30 \mathrm{~min}$ after i.v cadaverine injection, $n=4$ Unc5B $\mathrm{fl} / \mathrm{fl} ; \mathrm{Y} 949 \mathrm{~F}$ and $n=6$ Unc5BiECko;Y949F brains. Each dot represents one mouse. Western blot (g) and quantification (h) of brain protein extracts, $n=7$ Unc5Bfl/fl and $n=8$ Unc5BiECko brains. Each dot represents one mouse. One control mouse was set as 1. Immunofluorescence with the indicated antibodies (i) and quantification (j) of VE-cadherin coverage on P67 brain sections. Each dot is the mean of several images, $n=3$ Unc $5 B^{f l / f l}$ and $n=5$ Unc5BiECko brains. One control mouse was set as 1 . All data are shown as mean \pm SEM. NS non-significant. Two-sided Mann-Whitney $U$ test was performed for statistical analysis. Source data are provided as a Source Data file.

c). Internalization from the plasma membrane using this antibody is expected to prevent binding of all Unc5B ligands in vivo.

To generate a mAb that specifically blocked Netrin-1 binding without Unc5B internalization, we screened a human phagederived library against the entire rat Unc5B ECD and identified anti-Unc5B-3, a mAb that bound both human and rat Unc5B with high affinity (Fig. 6a-c) but did not induce Unc5B internalization nor its degradation in vivo (Supplementary Fig. 4d-f). I.v. injection of anti-Unc5B-3 for $15 \mathrm{~min}$ at $10 \mathrm{mg} / \mathrm{kg}$ followed by cardiac perfusion and immunolabelling using an antihuman IgG antibody revealed anti-Unc5B-3 binding to brain arteries and capillaries of $U n c 5 B^{f l f l}$, but no binding in the Unc5BiECko mice (Fig. 6d, e, Supplementary Fig. 5a), demonstrating specific binding of anti-Unc5B-3 to endothelial Unc5B. Anti-Unc5B-3 blocked Netrin-1-induced Src phosphorylation in brain ECs in vitro (Fig. 6f, g). To test effects on ligand binding in vivo, we injected control anti-Unc5B-1 or anti-Unc5B-3 i.v $(10 \mathrm{mg} / \mathrm{kg}$ for $1 \mathrm{~h})$, followed by Unc5B immunoprecipitation from brain lysates using a commercial antibody recognizing the Unc5B ECD. Western blotting revealed that anti-Unc5B-3 blocked Netrin-1 binding to Unc5B in vivo, while Robo4 and Flrt 2 could still interact with Unc5B (Fig. 6h, i).

To test if antibody-mediated Unc5B blockade could induce BBB leak, we injected i.v. CTRL or anti-Unc5B antibodies for $1 \mathrm{~h}$ in adult WT C57BL/6J mice, followed by i.v. injection of cadaverine $30 \mathrm{~min}$ before sacrifice and analysis (Fig. 6j). In mice treated with CTRL anti-Unc5B-1 or IgG Ab, there were no signs of $\mathrm{BBB}$ disruption and injected cadaverine remained confined inside brain vessels (Fig. 6k, l). In contrast, mice treated with antiUnc5B-3 or anti-Unc5B-2 for $1 \mathrm{~h}$ showed a significant leakage of injected cadaverine into the brain parenchyma (Fig. 6k, 1), demonstrating that blocking Netrin-1 binding to Unc5B is sufficient to open the BBB. Interestingly, i.v. injection of CTRL anti-Unc5B-1, anti-Unc5B-2 and -3 for $8 \mathrm{~h}$ prior to cadaverine injection for $30 \mathrm{~min}$ did not induce any BBB leakage (Fig. $6 \mathrm{~m}, \mathrm{n}$ ). Western blot against human IgG confirmed presence of all antibodies in the serum $1 \mathrm{~h}$ and $8 \mathrm{~h}$ after i.v. injection (Supplementary Fig. 4g), suggesting a transient BBB disrupting effect of anti-Unc5B-3 and -2 antibodies. The vascular barrier disrupting effect of anti-Unc5B-2 and -3 was specific to the brain, 

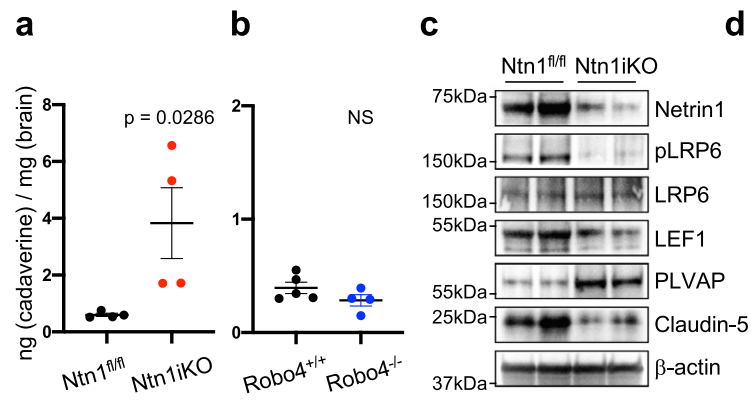

d

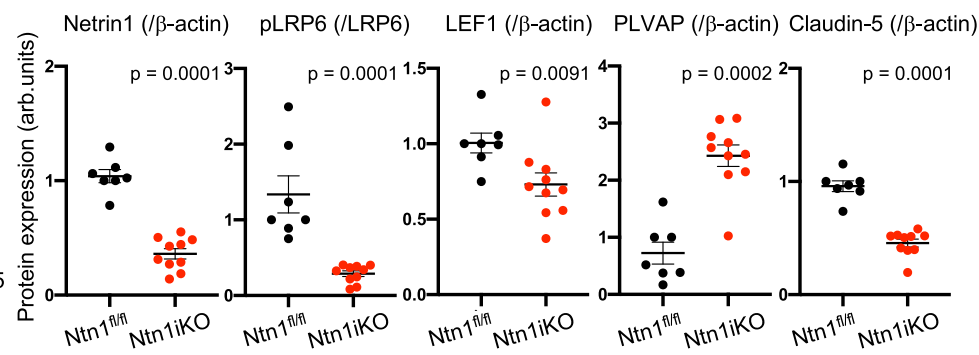

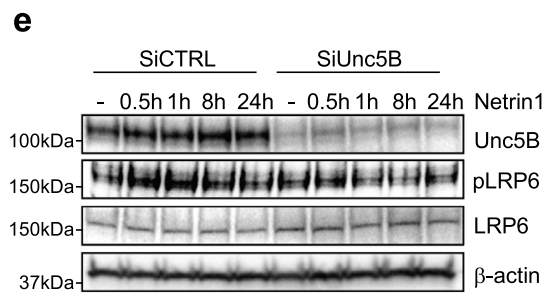

i

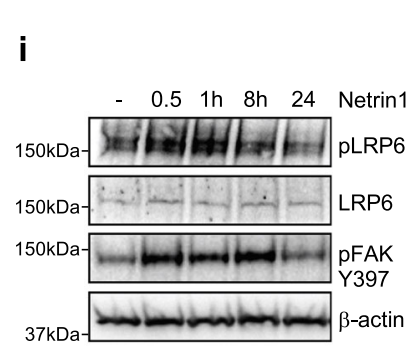

f

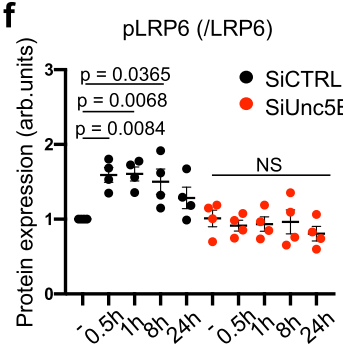

k

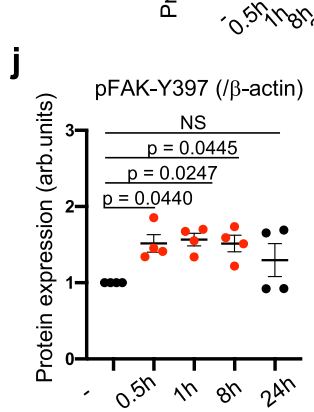

g
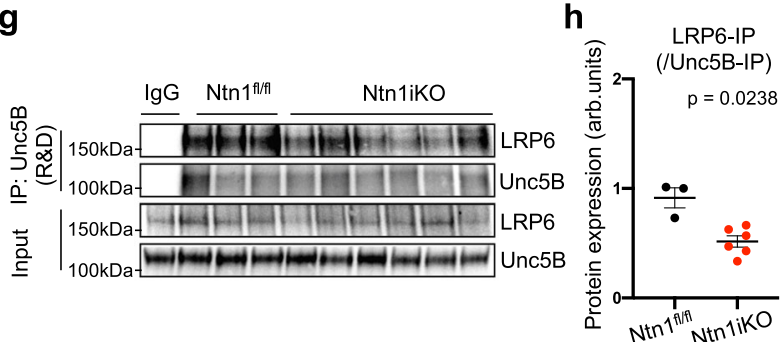

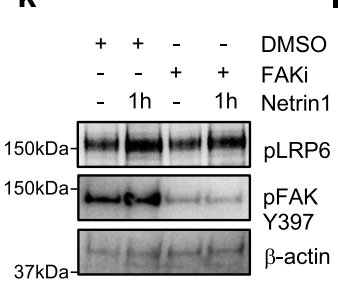

I

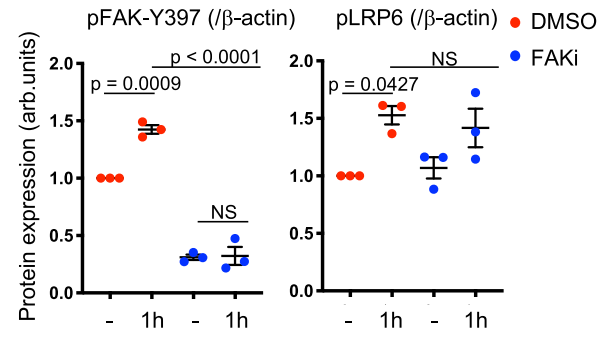

Fig. 5 Netrin-1 controls BBB integrity. a, b Quantification of cadaverine content in P67 brains, 30 min after i.v. cadaverine injection. Ntn1 gene deletion was induced by tamoxifen injection between P60 and P64, $n=4 \mathrm{Ntn} f^{f l / f l}, n=4 \mathrm{Ntn1iko,} n=5$ Robo4 ${ }^{+I+}$ and $n=4$ Robo4 ${ }^{+l-}$ brains. Each dot represents one mouse. Western blot (c) and quantification (d) of brain protein extracts, $n=7 \mathrm{Ntn} f^{f l} / \mathrm{fl}$ and $n=10 \mathrm{Ntn} 7 \mathrm{iko}$ brains. Each dot represents one mouse. One control mouse was set as 1 . Western blot (e) and quantification (f) of mouse brain ECs treated with scrambled CTRL or Unc5B siRNA for 48 h and treated with recombinant mouse Netrin-1 $(500 \mathrm{ng} / \mathrm{ml})$ or not $(-)$ for the indicated times. Each dot represents one independent experiment, $n=4$ independent experiment. $\mathbf{g}$ CTRL IgG or Unc5B immunoprecipitation of brain protein extracts and Western blot for LRP6. h quantification of LRP6 pulldown with Unc5B, $n=3 \mathrm{Ntn} 1 \mathrm{fl} / \mathrm{fl}$ and $n=6 \mathrm{Ntnliko}$ brains. Each dot represents one mouse. One control mouse was set as 1 . Western blot (i) and quantification ( $\mathbf{j}$ ) of mouse brain ECs treated with recombinant mouse Netrin-1 $(500 \mathrm{ng} / \mathrm{ml})$ or not $(-)$ for the indicated times. Each dot represents one independent experiment, $n=4$ independent experiment. Western blot (k) and quantification (I) of mouse brain ECs treated with CTRL DMSO or FAK inhibitor ( 5 uM) for 30 min followed by recombinant mouse Netrin-1 treatment $(500 \mathrm{ng} / \mathrm{ml})$ or not $(-)$ for $1 \mathrm{~h}$. Each dot represents one independent experiment, $n=3$ independent experiment (pFAK-Y397(/ $\beta$-actin): exact $p$-value between DMSO + Netrin1 $(1 \mathrm{~h})$ and FAKi + Netrin1 $(1 \mathrm{~h})=0.000031)$. All data are shown as mean \pm SEM. NS non-significant. Two-sided Mann-Whitney $U$ test was performed for statistical analysis between two groups. ANOVA followed by Bonferroni's multiple comparisons test was performed for statistical analysis between multiple groups. Source data are provided as a Source Data file.

as tracer leakage in other organs $1 \mathrm{~h}$ after i.v. injection was similar between controls and anti-Unc5B-2 or -3 treated mice (Fig. 6o-q).

Anti-Unc5B induced transient Wnt signaling inhibition. To assess anti-Unc5B bioavailability and vascular clearance, we i.v. injected anti-Unc5B-3 antibodies $(10 \mathrm{mg} / \mathrm{kg})$ for $1 \mathrm{~h}, 8 \mathrm{~h}$ or $24 \mathrm{~h}$ followed by immunolabeling with anti-human IgG antibodies (Fig. 7a). Anti-Unc5B-3 was detectable in the brain vasculature $1 \mathrm{~h}$ after injection, declined to low levels after $8 \mathrm{~h}$ and was undetectable $24 \mathrm{~h}$ after injection (Fig. $7 \mathrm{a}-\mathrm{c}$ ), demonstrating that the antibody was unstable and rapidly cleared from the brain vasculature.

Interestingly, the expression of $\mathrm{Wnt} / \beta$-catenin downstream targets varied in a similar time-dependent fashion. Claudin-5 immunostaining was downregulated $1 \mathrm{~h}$ after anti-Unc5B-3 injection and returned to basal levels after $8 \mathrm{~h}$, whereas PLVAP immunostaining was upregulated at 1 and $8 \mathrm{~h}$ after anti-Unc5B-3 injection and returned to low baseline levels after $24 \mathrm{~h}$ (Fig. 7a).
Claudin-5 and PLVAP expression changes occurred in brain capillaries but not in larger vessels ( $>10 \mathrm{um}$, Fig. 7a, Supplementary Fig. 5b). Two-photon live imaging through cranial windows confirmed BBB leak from brain capillaries $1 \mathrm{~h}$ after anti-Unc5B-3 i.v. injection (Supplementary Fig. 5c, d).

Unc5B immunoprecipitation showed that anti-Unc5B-3 treatment transiently disrupted the Unc5B/LRP6 interaction $1 \mathrm{~h}$ after injection (Fig. 7b, c). Western blot on brain protein lysates from mice treated with CTRL IgG or anti-Unc5B-3 confirmed transient downregulation of Claudin-5 at $1 \mathrm{~h}$, and upregulation of PLVAP at 1-8 h, and also showed transiently decreased LRP6 phosphorylation and decreased $\beta$-catenin protein levels $1-8 \mathrm{~h}$ after anti-Unc5B-3 injection that returned to baseline levels after $24 \mathrm{~h}$ (Fig. $7 \mathrm{~b}, \mathrm{c}$ ).

Size-selectivity of Unc5B mediated BBB opening. To characterize the size selectivity of $\mathrm{BBB}$ opening induced by genetic or antibody-mediated Unc5B inhibition, we injected fluorescent dextrans of increasing molecular weights into mice. We observed that both $10 \mathrm{kDa}$ and $40 \mathrm{kDa}$ dextrans had a higher permeability across 
a

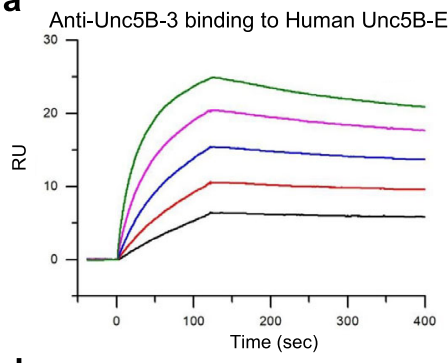

d

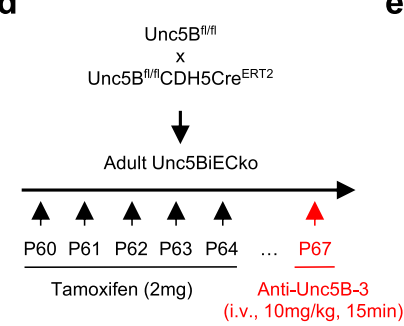

$$
\text { f }
$$

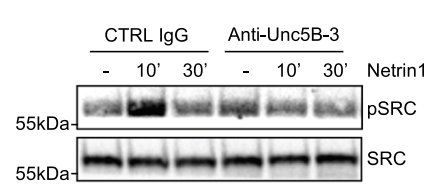

g
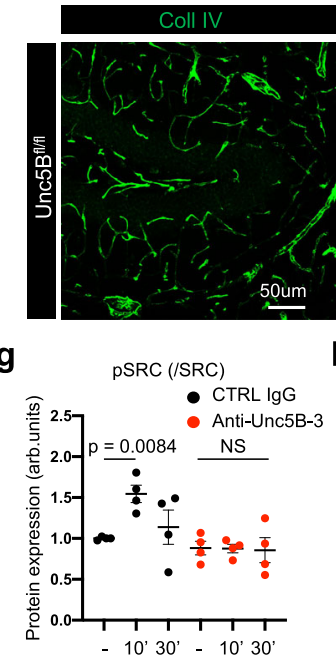

b

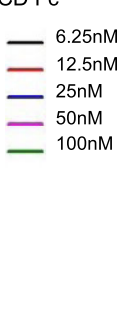

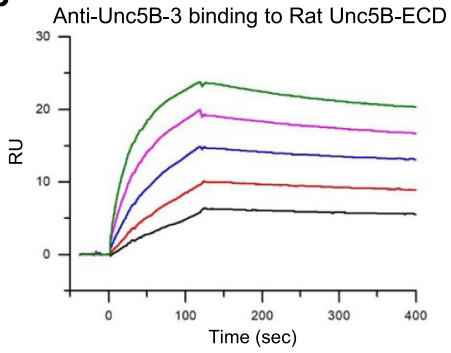

C

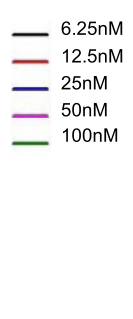

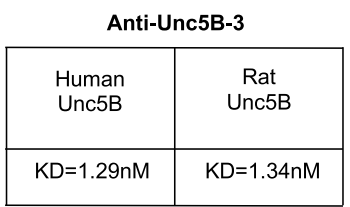
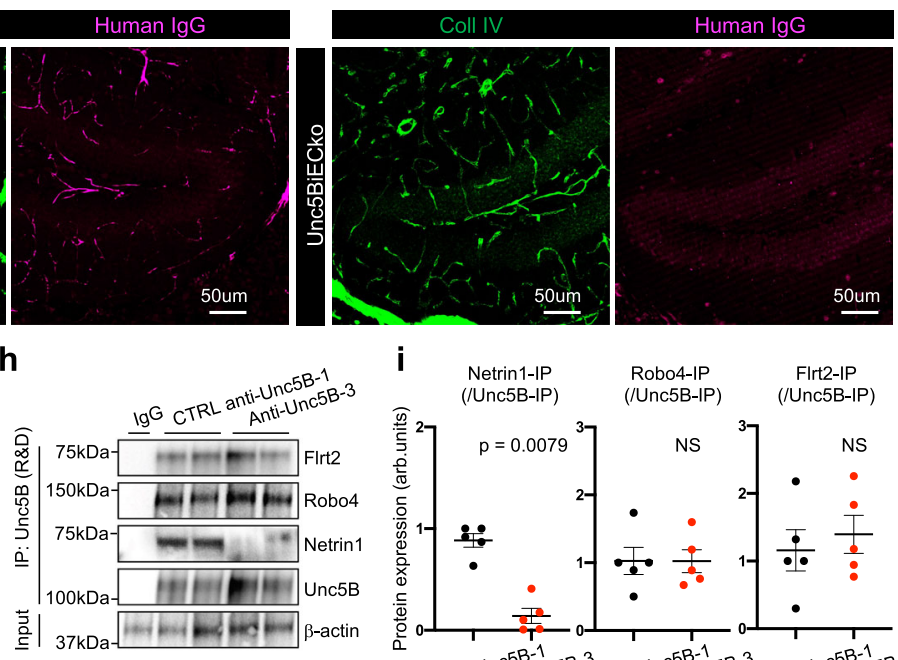

i $\begin{gathered}\text { Netrin1-IP } \\ \text { (/Unc5B-IP) }\end{gathered} \quad \begin{gathered}\text { Robo4-IP } \\ \text { (/Unc5B-IP) }\end{gathered} \quad \begin{gathered}\text { Firt2-IP } \\ \text { (/Unc5B-IP) }\end{gathered}$

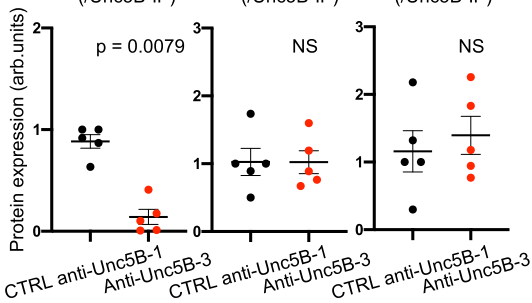

n

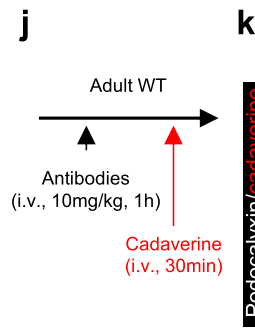

k
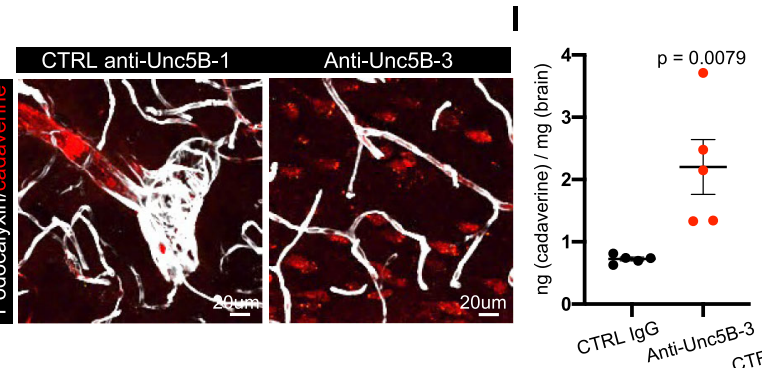

o

p

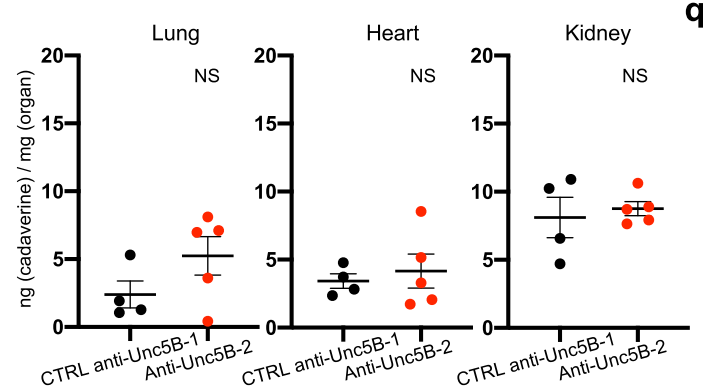

q

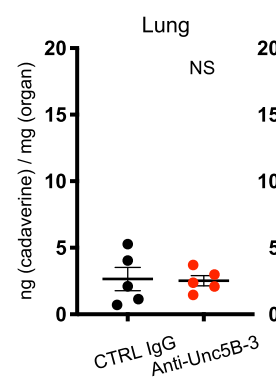

m
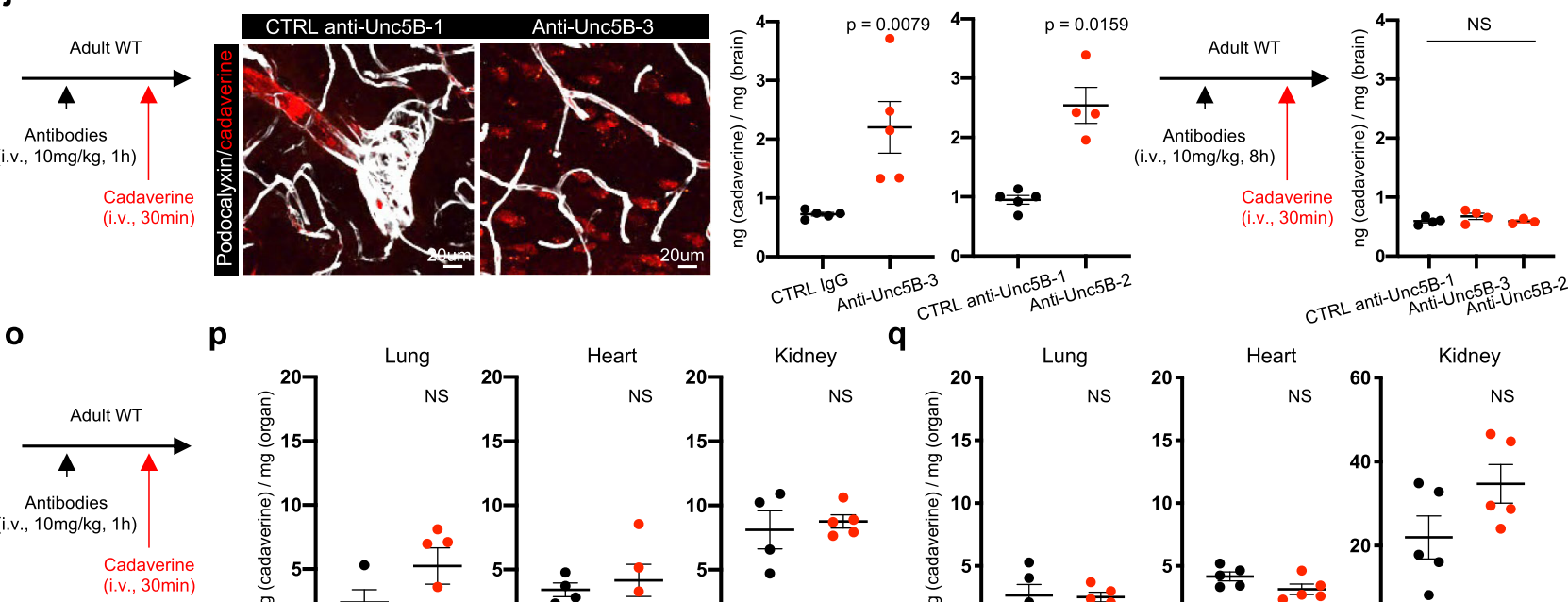

CTRL anti-Unc5 Anti-Ur

the BBB in Unc5BiECko brains and anti-Unc5B-2 and -3 treated animals compared to controls (Fig. 8a, b). By contrast, $70 \mathrm{kDa}$ dextran, IgG and fibrinogen did not cross the BBB (Fig. 8c, Supplementary Fig. 6a, b), indicating a size-selective defect of $\mathrm{BBB}$ leakage for proteins greater than $40 \mathrm{kDa}$. As observed for cadaverine, leakage of dextrans in peripheral organs was similar between groups (Fig. 8d-f). Interestingly, leakage of $40 \mathrm{kDa}$ dextran also remained increased in brains of Unc5BiECko;eGFP::Claudin-5 mice compared to Unc5BiECko mice (Supplementary Fig. 6c). Besides dextrans, anti-Unc5B-3 treatment also enhanced delivery of single-chain nanobodies across the BBB when compared to CTRL IgG (Fig. 8g), while nanobody extravasation in other organs such as lung, heart, kidney or skin remained similar (Supplementary Fig. 6d). Moreover, injection of anti-Unc5B-3 enhanced brain delivery of BDNF and induced phosphorylation and activation of its neuronal receptor Trk-B, while plasma BDNF levels remained similar to CTRL IgG injected mice (Fig. $8 \mathrm{~h}-\mathrm{j}$ ), indicating that bioactive molecules up to $40 \mathrm{kDa}$ can be delivered into the brain by this approach.

\section{Discussion}

Our data reveal endothelial Unc5B as a critical regulator of BBB integrity. We showed that adult mice deficient in endothelial Unc5B expression exhibited widespread BBB leakage from brain capillary ECs, which converted from a Claudin-5+/PLVAPBBB competent state to a leaky Claudin-5-/PLVAP+ state and displayed reduced expression of $\beta$-catenin and LEF1 (Supplementary Fig. 7). Combined heterozygous deletions of both Unc5B 
Fig. 6 Netrin-1 binding to Unc5B mediates BBB integrity. a, b Surface Plasmon Resonance measurements of anti-Unc5B-3 binding to human and rat Unc5B-ECD-Fc. c Dissociation constant for anti-Unc5B-3 binding to human and rat Unc5B. d Unc5B gene deletion strategy using tamoxifen injection. e Anti-Unc5B-3 was i.v. injected in P67 Unc5Bfl/fl or Unc5BiECko mice for 15 min. Mice were perfused and anti-Unc5B-3 binding was detected by immunofluorescence on brain sections using an anti-human lgG antibody, reproduced on $n=4$ Unc5 $B^{f / f l}$ and $n=3$ Unc5BiECko brain. Western-blot (f) and quantification (g) of ECs treated with CTRL IgG or anti-Unc5B-3 for $1 \mathrm{~h}$ followed by recombinant mouse Netrin-1 treatment (500 ng/ml) for $10 \mathrm{~min}$ or $30 \mathrm{~min}$. Each dot represents one independent experiment, $n=4$ independent experiment. $\mathbf{h}$ Unc5B immunoprecipitation with a commercial antibody (R\&D systems) of brain protein extracts from mice i.v injected with CTRL or anti-Unc5B-3 antibodies ( $1 \mathrm{~h}, 10 \mathrm{mg} / \mathrm{kg})$, and western blot with antibodies recognizing the indicated ligands. $\mathbf{i}$ Quantification of $\mathbf{h}, n=5$ CTRL anti-Unc5B-1 and $n=5$ anti-Unc5B-3 treated animals. Each dot represents one mouse. One control mouse was set as 1. j l.v. antibody injection strategy. $\mathbf{k}$ Immunofluorescence staining on brain sections from antibody-injected mice. I Quantification of brain cadaverine content, $n=5$ CTRL IgG, $n=5$ anti-Unc5B-3, $n=5$ CTRL anti-Unc5B-1 and $n=4$ anti-Unc5B-2 treated animals. Each dot represents one mouse. $\mathbf{m}$ I.v. antibody injection strategy. $\mathbf{n}$ Quantification of brain cadaverine content, $n=4$ CTRL anti-Unc5B-1, $n=4$ anti-Unc5B-3 and $n=3$ anti-Unc5B-2 treated animals. Each dot represents one mouse. o I.v. antibody injection strategy. p, q Quantification of cadaverine content in peripheral organs, $n=4$ CTRL anti-Unc5B-1, $n=5$ anti-Unc5B-2, $n=5$ CTRL IgG and $n=5$ anti-Unc5B-3 treated animals. Each dot represents one mouse. All data are shown as mean \pm SEM. NS non-significant. Two-sided Mann-Whitney $U$ test was performed for statistical analysis between two groups. ANOVA followed by Bonferroni's multiple comparisons test was performed for statistical analysis between multiple groups. Source data are provided as a Source Data file.

a

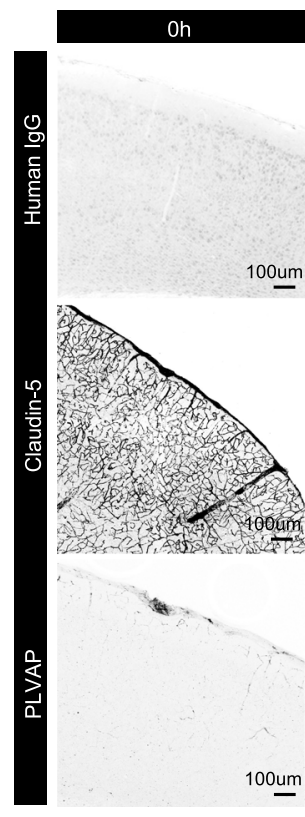

C $\quad$ LRP6-IP (Unc5B-IP)

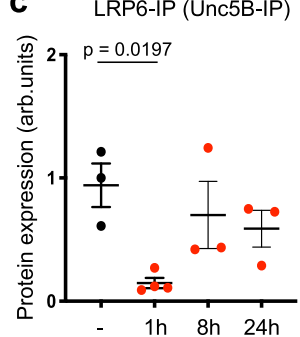
Anti-Unc5B-3: $1 \mathrm{~h}$ Anti-Unc5B-3: 8h Anti-Unc5B-3: 24h
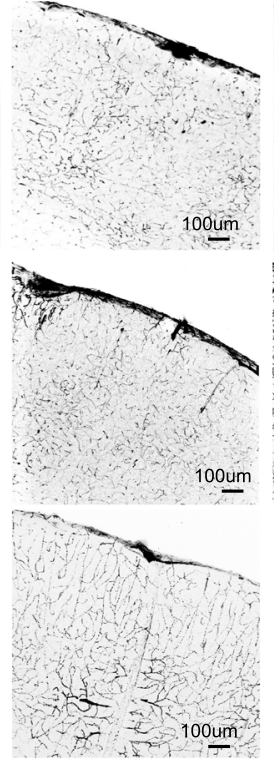

pLRP6 (/LRP6)

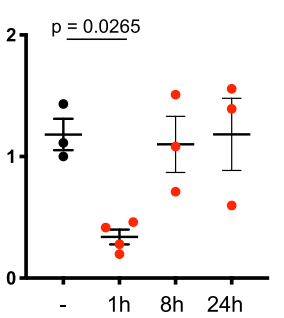

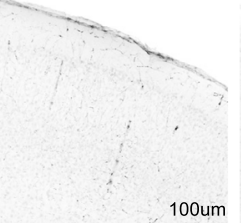

${ }_{T}^{100 u m}$
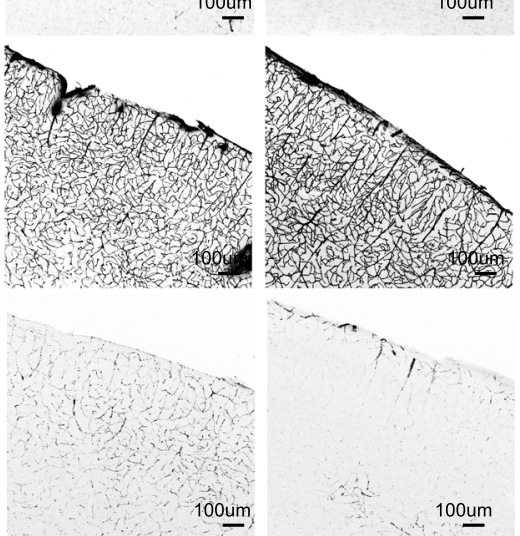

100um
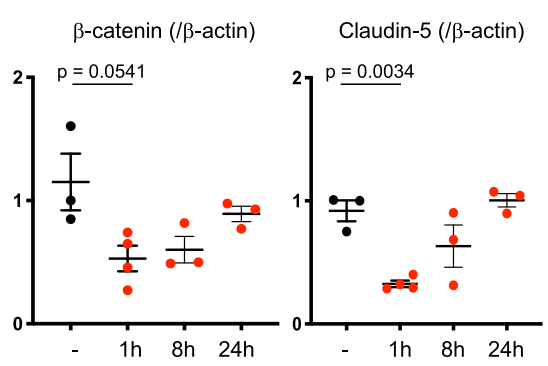

b

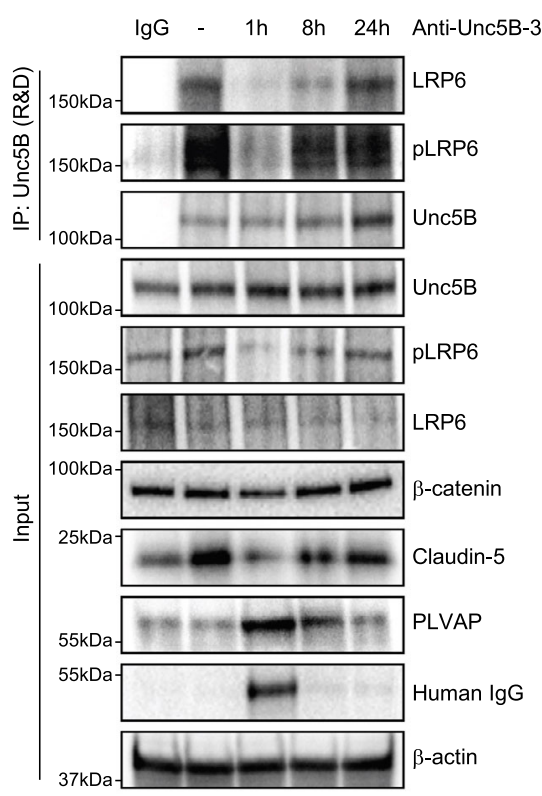

PLVAP (/ß-actin)

Human IgG (/ $\beta$-actin)

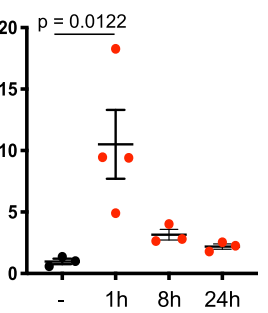

Fig. 7 Wnt / $\beta$-catenin signaling regulation by Unc5B-blocking antibodies. a Immunofluorescence staining of Human IgG, Claudin-5 and PLVAP and confocal imaging on brain sections from mice i.v. injected with anti-Unc5B-3 (10 mg/kg) for $1 \mathrm{~h}, 8 \mathrm{~h}$ or $24 \mathrm{~h}$, reproduced on $n=3$ untreated brains, $n=4$ anti-Unc5B-3 treated brains for $1 \mathrm{~h}, n=3$ anti-Unc5B-3 treated brains for 8 and $n=3$ anti-Unc5B-3 treated brains for $24 \mathrm{~h}$. b CTRL IgG or Unc5B immunoprecipitation of brain protein extracts from mice i.v. injected with anti-Unc5B-3 and protein quantification (c), $n=3$ control IgG treated brains, $n=4$ anti-Unc5B-3 treated brains for $1 \mathrm{~h}$ and $n=3$ anti-Unc5B-3 treated brains for 8 and $n=3$ anti-Unc5B-3 treated brains for $24 \mathrm{~h}$. Each dot represents one mouse. One control mouse was set as 1 . All data are shown as mean \pm SEM. NS non-significant. ANOVA followed by Bonferroni's multiple comparisons test was performed for statistical analysis between multiple groups. Source data are provided as a Source Data file.

and $\beta$-catenin induced BBB leak of cadaverine, while mice carrying single heterozygous deletions in either Unc5B or $\beta$-catenin displayed an intact BBB; and endothelial-specific $\beta$-catenin overexpression in Unc5BiECko mice increased Claudin-5 and LEF1 expression, while suppressing PLVAP and cadaverine leak, together supporting that Unc5B maintains BBB integrity by functionally interacting with Wnt signaling.

Loss of endothelial Unc5B induced cadaverine leakage across the BBB in a widespread but non-uniform manner, in that caudal and ventral brain regions were more affected by loss of Unc5B 
a

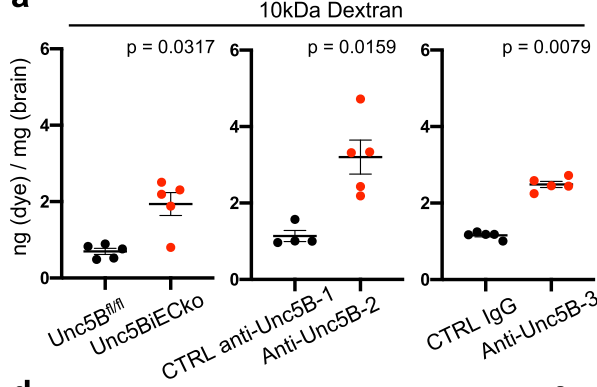

b

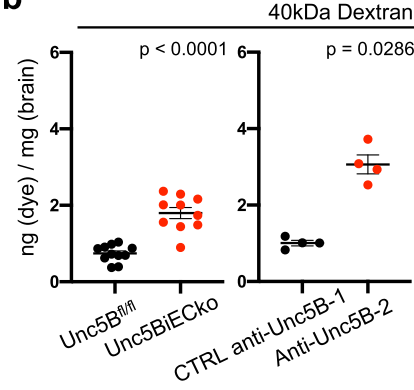

C

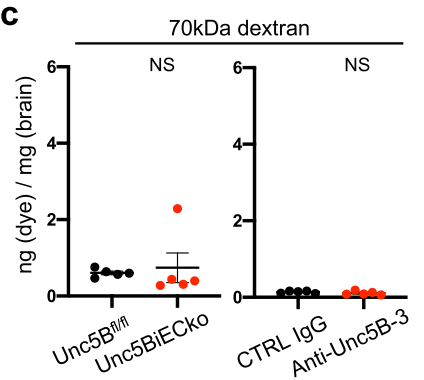

d

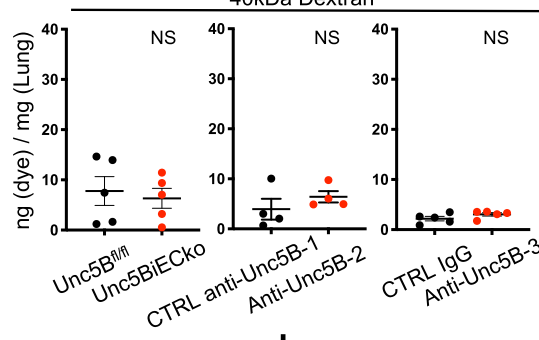

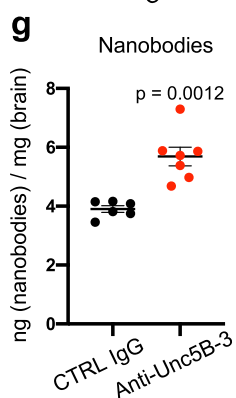

h BDNF (brain) e

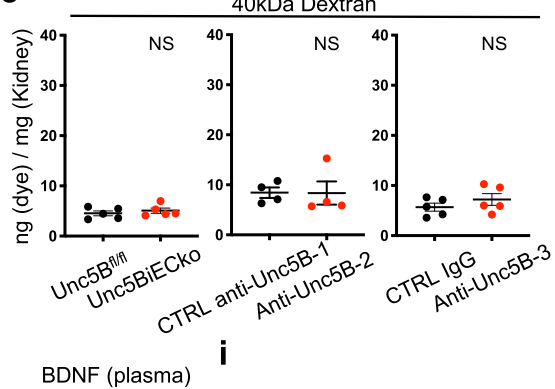
f

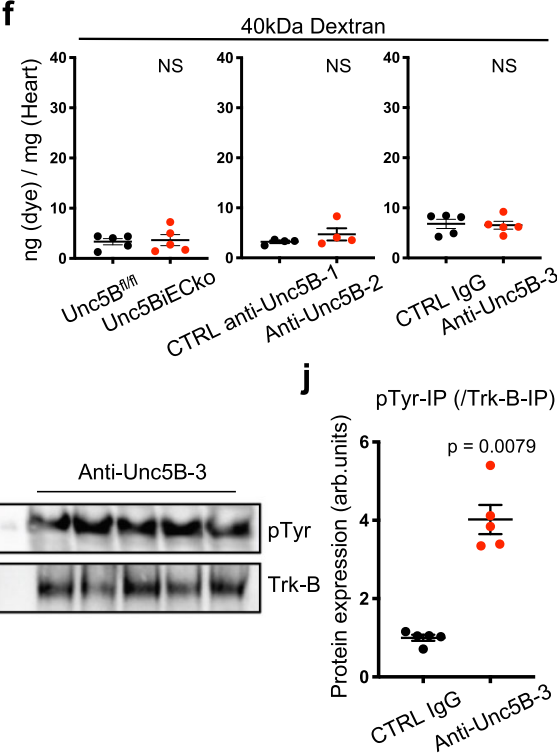

Fig. 8 Size-selectivity of anti-Unc5B induced BBB opening. Quantification of $10 \mathrm{kDa}$ (a) $40 \mathrm{kDa}$ (b) and $70 \mathrm{kDa}$ (c) dextran content in P67 brains. Unc5B ${ }^{\mathrm{fl} / \mathrm{fl}}$ and Unc5BiECko mice were injected with tamoxifen between P60-P64. CTRL anti-Unc5B-1, CTRL IgG, anti-Unc5B-2 and anti-Unc5B-3 antibodies were i.v. injected $(10 \mathrm{mg} / \mathrm{kg})$ for $1 \mathrm{~h}$. Dextran was i.v. injected for $30 \mathrm{~min}$. Quantification of $10 \mathrm{kDa}$ dextran was performed on $n=5 \mathrm{Unc} 5 \mathrm{~B}^{\mathrm{fl} / \mathrm{fl}}$ and $n=5$ Unc5BiECko brains, $n=4$ CTRL anti-Unc5B-1, $n=5$ anti-Unc5B-2, $n=5$ CTRL IgG and $n=5$ anti-Unc5B-3 treated brains. Quantification of $40 \mathrm{kDa}$ dextran was performed on $n=11$ Unc5B $\mathrm{B}^{f / f l}$ and $n=10$ Unc5BiECko brains (exact $p$-value $=0.000040$ ), $n=4$ CTRL anti-Unc5B-1, $n=4$ anti-Unc5B-2, $n=5$ CTRL IgG and $n=5$ anti-Unc5B-3 treated brains. Quantification of $70 \mathrm{kDa}$ dextran was performed on $n=5$ Unc5Bfl/fl and $n=5$ Unc5BiECko brains, $n=5 \mathrm{CTRL}$ IgG and $n=5$ anti-Unc5B-3 treated brains. Each dot represents one mouse. d-f Quantification of $40 \mathrm{kDa}$ dextran content in P67 organs, $n=5$ Unc5B $f / / f l$ and $n=5$ Unc5BiECko brains, $n=4$ CTRL anti-Unc5B-1, $n=4$ anti-Unc5B-2, $n=5$ CTRL IgG and $n=5$ anti-Unc5B-3 treated brains. Each dot represents one mouse. $\mathbf{g}$ Quantification of P67 brain nanobody content $1 \mathrm{~h}$ after i.v CTRL $\mathrm{ggG}$ or anti-Unc5B-3 injection (10 mg/kg) and 30 min after i.v nanobody injection, $n=6$ CTRL IgG and $n=7$ anti-Unc5B-3 treated brains. Each dot represents one mouse. $\mathbf{h}$ Quantification of P67 brain and plasma BDNF concentration $1 \mathrm{~h}$ after i.v CTRL IgG or anti-Unc5B-3 injection (10 mg/ $/ \mathrm{kg}$ ) and $30 \mathrm{~min}$ in after i.v BDNF injection, $n=7$ CTRL IgG and $n=7$ anti-Unc5B-3 treated brains. Each dot represents one mouse. Trk-B immunoprecipitation of brain protein extracts from mice i.v. injected with CTRL IgG or anti-Unc5B-3 antibodies (10 mg/ $\mathrm{kg}$ ) for $1 \mathrm{~h}(\mathbf{i})$ and quantification of phospho-tyrosine pulldown (j), $n=5$ CTRL IgG and $n=5$ anti-Unc5B-3 treated brains. Each dot represents one mouse. One control mouse was set as 1 . All data are shown as mean \pm SEM. NS: non-significant. Two-sided Mann-Whitney $U$ test was performed for statistical analysis between two groups. Source data are provided as a Source Data file.

than anterior and dorsal ones. This pattern of BBB disruption is roughly similar to the one observed in mice carrying inducible endothelial deletions of $\beta$-catenin ${ }^{21,24}$. We note that BBB leakage did not strictly correlate with Unc5B expression levels. Firstly, all cortical areas expressed endothelial Unc5B, but not all cortical areas were leaky in its absence. Secondly, Unc5B was detected in arteries and in capillaries, but only capillaries converted to a Claudin-5 negative, PLVAP-positive state in Unc5BiECko brains. The reasons for these region- and vessel segment-specific differences remain to be further investigated, but they may relate to $\beta$ - catenin levels.

The specific Wnt ligands and receptors that maintain the BBB differ in a region-specific manner in the CNS, with cerebellum BBB utilizing Norrin signaling through LRP5, Fzd4 and TSPAN12, while cortex relies on Wnt7a/b signaling through LRP5/6, Fzd4, GPR124 and RECK ${ }^{16,18-21,24}$. Intercross between $N d p$ (Norrin) and Wnt7a mutants revealed that, with loss of additional $W n t 7 a$ alleles in the $N d p$ KO background, there was a stepwise increase in the conversion of cerebellar ECs to a permeable state ${ }^{21}$. These and other genetic interaction studies between Wnt signaling components have suggested that a critical $\beta$-catenin threshold is required to maintain BBB ECs in a Clau-

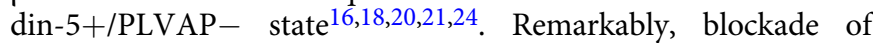
Unc5B function affected both the cortex, cerebellum and other brain regions, suggesting that Unc5B may be an upstream regulator of both Wnt and Norrin signaling complexes at the BBB.

Mechanistically, we identify Netrin-1 as an Unc5B activating ligand required for $\mathrm{BBB}$ maintenance. Mice carrying global inducible deletions of the Unc5B ligand Netrin-1, and i.v. delivery of Unc5B mAbs that specifically block Netrin-1 binding to Unc5B (anti-Unc5B-3), or that induce receptor internalization (antiUnc5B-2), all led to BBB breakdown and reduced $W n t / \beta$-catenin signaling, while Robo4 $-/-$ mice displayed an intact BBB. These data support that Netrin-1 binding to Unc5B maintains BBB 
integrity and Wnt signaling activation in CNS ECs. They confirm previous observations of Netrin's BBB-protective effects ${ }^{52,53}$ and extend them in several ways. Podjaski et al. ${ }^{52}$ showed that BBB junctional integrity was compromised in neonatal global Ntn1 ko mice, however the neonatal lethality of these mice precluded analysis of the BBB at later developmental stages, and the relevant Netrin-1 receptor was not identified in that study. We extend these findings by showing that global inducible deletion of Ntn1 in P60 mice induced BBB cadaverine leak, demonstrating that maintenance of adult BBB integrity requires Netrin-1. Furthermore, we identify the Unc5B receptor as the signal transducer on ECs, and we reveal canonical Wnt signaling as an effector pathway. Further experiments are required to determine the source of Netrin-1 mediating EC Unc5B signaling. Single cell RNA sequencing studies indicate that Netrin-1 is expressed in adult brain pericytes, fibroblasts, astrocytes and in $\mathrm{ECs}^{49}$. Conditional Ntn1 deletion in astrocytes affects the BBB $^{53}$, and Netrin-1 is upregulated in ECs upon inflammatory signaling ${ }^{52}$, therefore multiple cellular sources and environmental modulations of Netrin-1 expression could contribute to BBB integrity. Interestingly, serum Netrin-1 levels increased in patients with neuroinflammatory multiple sclerosis or type 2 diabetes ${ }^{52,54}$. Therefore, circulating Netrin-1 levels could be an important gatekeeper of BBB integrity, protecting the CNS and limiting BBB disruption during inflammatory conditions.

Mechanistically, the data show that Netrin-1 binding to Unc5B promotes LRP6 phosphorylation, and that the Unc5B intracellular UPA domain is required for complex formation between Unc5B and LRP6. Based on the crystal structure of the Unc5B $\mathrm{ICD}^{33}$, we speculate that ligand-induced conformational changes in the Unc5B ICD expose the UPA domain and enable its interaction with LRP6. One possibility is that the UPA domain may induce LRP6 phosphorylation through recruitment of kinases. Recent studies in naïve pluripotent embryonic stem cells showed that Netrin-1 binding to Unc5B induced FAK-mediated phosphorylation of GSK3a/ $\beta$, a kinase implicated in LRP6 activation $^{51}$, however our data show that Netrin-1 regulates LRP6 phosphorylation in brain ECs independently of FAK.

I.v. injected anti-Unc5B antibodies bound to the EC lumen in controls but not in Unc5BiECko brains, demonstrating Unc5B expression at the luminal side of brain ECs, which could be activated by Netrin-1 in the bloodstream. A critical question to be addressed in future work is how luminal Unc5B activates CNS Wnt signaling, which is believed to occur mainly at the abluminal side of CNS capillaries driven by neural progenitors or gliaderived WNTs and Norrin. Netrin-1-binding to luminal Unc5B could enhance Wnt/ $\beta$-catenin signaling in endosomes or at cellcell junctions, thereby facilitating subthreshold abluminal WNT $\mathrm{BBB}$ signaling effects.

Previous studies had speculated that transient Wnt signaling inhibition could be used to open the BBB "on-demand" for drug delivery into the diseased $\mathrm{CNS}^{20,24}$, but the means to inhibit Wnt signaling in a CNS specific manner were not available. We demonstrated that antibody-mediated Unc5B blockade caused a transient loss of Wnt $/ \beta$-catenin signaling and BBB breakdown for $1 \mathrm{~h}$ to $8 \mathrm{~h}$ followed by neurovascular barrier resealing, and allowed delivery of tracers up to $40 \mathrm{kDa}$ into the adult CNS. The size selectivity of $\mathrm{BBB}$ opening is compatible with delivery of chemotherapeutics and of bioactive molecules such as nanobodies and growth factors in the specific regions of the brain where Unc5B regulates $B B B$ integrity. Anti-Unc5B mAbs could therefore synergize with existing therapies such as focused ultrasound/ microbubble approaches ${ }^{5-58}$ and offer a therapeutic perspective for treatment of various human neurological disorders. Conversely, our data also raise the possibility that intravenous injection of Netrin-1 could increase Wnt $/ \beta$-catenin signaling at the BBB and repair CNS endothelial barrier breakdown in conditions such as multiple sclerosis or ischemic stroke where Netrin-1, Unc5B and several Wnt/ $\beta$-catenin target genes are downregulated $^{59}$.

\section{Methods}

Mouse models. All protocols and experimental procedures were approved by the Yale University Institutional Animal Care and Use Committee (IACUC). Animals were housed at $20-24^{\circ} \mathrm{C}$, with $30-70 \%$ humidity under a $12 \mathrm{~h}$ light-dark cycle. Generation of the targeted $U n c 5 b$ allele was performed by homologous recombination in R1 ES cells. Correctly targeted cells were identified by Southern blot hybridization and injected into B6J blastocysts to generate Unc5bneo/+ mice. To remove the neo cassette, Unc5bneo/+ mice were mated to B6.129S4-Gt(ROSA) 26Sortm1(FLP1)Dym/RainJ mice (The Jackson Laboratory, stock \#009086). Mice were backcrossed to B6J mice for ten generations. Unc5B fl/fl $(\mathrm{B} 6-U n c 5 b<t m 1$ (flox) Slac/Slac) mice were then bred with Cdh5Cre ERT2 mice $^{35}$ or PDGFR $\beta C r e^{\text {ERT237. }}$. TCF/LEF:H2B-GFP mice, eGFP::Claudin-5 mice, Y949F mice, $\beta$ catenin GOF

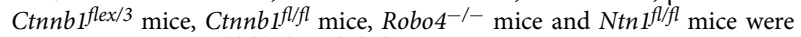
described previously $29,39,43,44,46,48,50$. Gene deletion was induced by injection of tamoxifen (Sigma T5648) diluted in corn oil (Sigma C8267). Postnatal gene deletion was induced by 3 injections of 100ug of tamoxifen at P0, P1 and P2; whereas adult gene deletion was induced by 5 injections of $2 \mathrm{mg}$ of tamoxifen from P60 to P64. Animals from both sexes were used.

Cell culture. C57BL/6 Mouse Primary Brain Microvascular Endothelial Cells were purchased from Cell Biologics (C57-6023). Cells were cultured in Dulbecco's Modified Eagle's Medium (DMEM) high glucose (Thermo Fisher Scientific, 11965092) supplemented with 10\% fetal bovine serum (FBS) and $1 \%$ Penicillin Streptomycin at $37^{\circ} \mathrm{C}$ and $5 \% \mathrm{CO}_{2}$ and split when confluent using Trypsin-EDTA $(0.05 \%)$ (Life Technologies, 25300054). When indicated, cells were treated with recombinant Mouse Netrin-1 Protein (R\&D, 1109-N1-025) at $500 \mathrm{ng} / \mathrm{ml}$, or with $5 \mathrm{uM}$ of FAK inhibitor (GSK2256098, Selleckem S8523).

Western-blot. Brains were dissected and frozen in liquid nitrogen. They were lysed in RIPA buffer (Research products, R26200-250.0) supplemented with protease and phosphatase inhibitor cocktails (Roche, 11836170001 and 4906845001) using a Tissue-Lyser (5 times $5 \mathrm{~min}$ at 30 shakes/second). For western blot on cell culture, cells were washed with PBS and lysed in RIPA buffer with protease and phosphatase inhibitors cocktails. All protein lysates were then centrifuged $15 \mathrm{~min}$ at $16,100 \times \mathrm{g}$ at $4^{\circ} \mathrm{C}$ and supernatants were isolated. Protein concentrations were quantified by BCA assay (Thermo Scientific, 23225) according to the manufacturer's instructions. 30 ug of protein were diluted in Laemmli buffer (Bio-Rad, 1610747 ) boiled at $95^{\circ} \mathrm{C}$ for $5 \mathrm{~min}$ and loaded in $4-15 \%$ acrylamide gels (Bio-Rad, 5678084). After electrophoresis, proteins were transferred on a polyvinylidene difluoride (PVDF) membrane and incubated in TBS $0.1 \%$ Tween supplemented with $5 \%$ BSA for $1 \mathrm{~h}$ to block non-specific binding. The following antibodies were incubated overnight at $4{ }^{\circ} \mathrm{C}$ : Unc5B (Cell Signaling, 13851S, dilution: 1/1000), Robo4 (Invitrogen, 20221-1-AP, dilution: 1/300), Flrt2 (Novus bio, NBP2-43653, dilution: 1/500), Netrin-1 (R\&D, AF1109, dilution: 1/500), Claudin-5 (Invitrogen, 35-2500, dilution: 1/1000), PLVAP (BD biosciences, 550563, dilution: 1/200), PDGFR $\beta$ (Cell Signaling, 3169S, dilution: 1/1000), GFAP (Millipore, MAB360, dilution: 1/1000), VEGFR2 Y949 (Cell Signaling, 4991S, dilution: 1/500), VEGFR2 Y1173 (Cell Signaling, 2478S, dilution: 1/500), pLRP6 (Cell Signaling, 2568S, dilution: 1/300), LRP6 (Cell Signaling, 3395S, dilution: 1/500), $\beta$ catenin (Cell Signaling, 8480S, dilution: 1/2000), LEF1 (Cell Signaling, 2230S, dilution: 1/1000), ZO1 (Invitrogen, 61-7300, dilution: 1/2000), Occludin (Invitrogen, 33-1500, dilution: 1/1000), Caveolin-1 (Cell Signaling, 3238S, dilution: 1/2000), VE-cadherin (BD Pharmingen, 555289, dilution: 1/500) and ßactin (Sigma, A1978, dilution: $1 / 5000$ ). Then, membranes were washed $4 \times 10 \mathrm{~min}$ in TBS $0.1 \%$ Tween and incubated with one of the following peroxidase-conjugated secondary antibodies diluted $1 / 5000$ in TBS $0.1 \%$ Tween supplemented with 5\%BSA for $2 \mathrm{~h}$ at room temperature: horse anti-mouse $\operatorname{IgG}(\mathrm{H}+\mathrm{L})$ (Vector laboratories, PI-2000), goat anti-rabbit $\operatorname{IgG}(\mathrm{H}+\mathrm{L})$ (Vector laboratories, PI-1000), goat anti-rat $\operatorname{IgG}(\mathrm{H}+\mathrm{L})$ (Vector laboratories, PI-9400), horse anti-goat $\operatorname{IgG}(\mathrm{H}+\mathrm{L})$ (Vector laboratories, PI9500). After 4 x 10 min wash, western blot bands were acquired using ECL western blotting system (Thermo Scientific, 32106) or west femto maximum sensitivity substrate (Thermo Scientific, 34095) on a Biorad Gel Doc EQ System with Universal Hood II imaging system equipped with Image Lab software. See Supplementary Figs. 8-11 for the uncropped immunoblots.

Immunoprecipitation. Pierce ${ }^{\mathrm{Ts}}$ protein A/G magnetic beads (Thermo fischer, 88802 ) were washed 5 times $10 \mathrm{~min}$ with RIPA buffer. 300 ug of protein lysate were diluted in $1 \mathrm{ml}$ of RIPA buffer containing protease and phosphatase inhibitors and were incubated with $30 \mathrm{ul}$ of A/G magnetic beads for $1 \mathrm{~h}$ at $4{ }^{\circ} \mathrm{C}$ under gentle rotation. Protein lysates were harvested using a magnetic separator (Invitrogen) and were incubated overnight at $4{ }^{\circ} \mathrm{C}$ under gentle rotation with 10 ug of Unc5B antibody (R\&D, AF1006) or control IgG. The next day, $40 \mathrm{ul}$ of protein A/G magnetic beads were added to each protein lysate for $2 \mathrm{~h}$ at $4{ }^{\circ} \mathrm{C}$ under gentle 
rotation. Beads were then isolated using a magnetic separator and washed 5 times with RIPA buffer. After the last wash, supernatants were removed and beads were resuspended in 40ul of Laemmli buffer (Bio-Rad, 1610747), boiled at $95^{\circ} \mathrm{C}$ for 5 min and loaded onto $4-15 \%$ gradient acrylamide gels. Western blotting was performed as described above.

Immunostaining. Brains were collected and placed in 3.7\% formaldehyde overnight at $4{ }^{\circ} \mathrm{C}$. Brains were then washed 3 times 10 min with TNT buffer (for $100 \mathrm{ml}$ $10 \mathrm{ml}$ Tris $1 \mathrm{M} \mathrm{pH7}, 4,3 \mathrm{ml} \mathrm{NaCl} 5 \mathrm{M}, 500 \mathrm{ul}$ Triton X-100) and embedded in $2 \%$ agarose. $100 \mathrm{um}$ sections were prepared using a Leica VT 1000S vibratome and placed in TNTB buffer (TNT buffer supplemented with 5\% donkey serum) for $24 \mathrm{~h}$ at $4{ }^{\circ} \mathrm{C}$. Primary antibodies were diluted in TNTB and placed for $48 \mathrm{~h}$ at $4{ }^{\circ} \mathrm{C}$ under gentle agitation. Then, sections were washed 5 times 30 min with TNT buffer and incubated for $24 \mathrm{~h}$ at $4{ }^{\circ} \mathrm{C}$ with secondary antibodies diluted in TNTB buffer. After $5 \times 30$ min wash with TNT, sections were mounted using DAKO mounting medium (Agilent, S302380-2).

For mouse brain endothelial cell immunostaining, cells were seeded on $18 \mathrm{~mm}$ glass coverslips (Fischer Scientific, 12542A). When confluent, cells were washed with PBS and fixed with $3.7 \%$ formaldehyde for $10 \mathrm{~min}$. Cells were washed $3 \mathrm{x}$ 5 min with PBS and were incubated with $0.2 \%$ TritonX100 diluted in PBS for an additional $10 \mathrm{~min}$, washed 3 times and incubated with blocking solution (2\%BSA, $3 \%$ Donkey serum diluted in PBS) for $1 \mathrm{~h}$ at room temperature. Primary antibodies were then diluted in blocking solution and incubated on coverslips overnight at $4^{\circ} \mathrm{C}$. After $3 \times 5$ min washes, secondary antibodies diluted in blocking buffer were incubated on coverslips for $2 \mathrm{~h}$ at room temperature. Coverslips were then washed $3 \mathrm{x} 5$ min with PBS and mounted using DAKO mounting medium.

The following antibodies were used: Podocalyxin (RD, AF1556, dilution: 1/400), Unc5B (Cell signaling, 13851S, dilution: 1/200), Claudin-5-GFP (Invitrogen, 352588, dilution: 1/200), PLVAP (BD biosciences, 550563, dilution: 1/100), LEF1 (Cell Signaling, 2230S, dilution: 1/200), GFAP (Millipore, MAB360, dilution: 1/ 400), Aquaporin-4 (Millipore, AB3068, dilution: 1/400), PDGFR $\beta$ (Cell Signaling, 3169S, dilution: 1/400), Endomucin (Hycult biotech, HM1108, dilution: 1/400), fibrinogen (DAKO, A0080, dilution: 1/400), CD13 (BD Biosciences, 558744, dilution: 1/200), VE-cadherin (BD Pharmingen, 555289, dilution: 1/200), DAPI (Thermo Fischer, 62248, dilution: 1/2000). All corresponding secondary antibodies were purchased from Invitrogen as donkey anti-primary antibody coupled with either Alexa Fluor 488, 568 or 647 and were diluted 1/200.

Small interfering RNA knockdown experiments. Cells were transiently transfected with siRNA (Dharmacon). ON-TARGETplus Mouse Unc5b siRNAs (SMARTpool, L-050737-01-0005) were used for Unc5B gene deletion. Transfection was performed using lipofectamine RNAi max (Invitrogen, 13778-075) according to the manufacturer's instruction with siRNA at a final concentration of $25 \mathrm{pmol}$ in OptiMem for $8 \mathrm{~h}$. After transfection, cells were washed with PBS and fresh complete media was added for $48 \mathrm{~h}$.

Mouse lung endothelial cell isolation. Mouse lungs were collected and minced into small pieces. Lungs were incubated in digestion buffer $(5 \mathrm{ml}$ of DMEM supplemented with $5 \mathrm{mg}$ of collagenase I (Worthington LS004196), 10ul of $1 \mathrm{M} \mathrm{Ca}^{2+}$ and $10 \mathrm{ul}$ of $1 \mathrm{M} \mathrm{Mg}^{2+}$ ) for $1 \mathrm{~h} \mathrm{at} 37^{\circ} \mathrm{C}$ with shaking every $10 \mathrm{~min}$. Once fully lysed, lung lysates were filtered through a 40um cell strainer (Falcon, 352340) into a solution of $3 \mathrm{ml} \mathrm{FBS}$. Samples were centrifuged for $10 \mathrm{~min}$ at $200 \mathrm{~g}$, and pellets were resuspended in PBS 0.1\%BSA. In the meantime, rat anti-mouse CD31 (BD Pharmingen, 553370) was incubated with sheep anti-rat IgG magnetic dynabeads (Invitrogen, 11035) in a solution of sterile PBS 0.1\%BSA (120ul of beads, $24 \mathrm{ul}$ of antibodies in $12 \mathrm{ml}$ PBS $0.1 \% \mathrm{BSA}$ ). Solutions were place under gentle rotation at room temperature for $2 \mathrm{~h}$ to allow proper coupling of antibodies and beads. Coupled beads were next isolated using a magnetic separator and incubated in the resuspended lung lysate for $30 \mathrm{~min}$. After 5 washes with PBS $0.1 \% \mathrm{BSA}$, beads were separated using magnetic separator and seeded in $60 \mathrm{~mm}$ dishes containing mouse lung endothelial cell media (DMEM high glucose, 20\%FBS, 1\% Penicillin Streptomycin, $2 \%$ mitogen (Alta Aesar BT203). Purified endothelial cells were cultured at $37^{\circ} \mathrm{C}$ and $5 \% \mathrm{CO}_{2}$ until confluence was reached, and then harvested.

Quantitative real-time PCR analysis. mRNA was isolated using Trizol reagent (Life Technologies, 15596018) according to the manufacturer's instructions and quantified RNA concentrations using nanodrop 2000 (Thermo Scientific). $300 \mathrm{ng}$ of RNA were reverse transcribed into cDNA using iScript cDNA synthesis kit (Biorad, 170-8891). Real-time qPCR was then performed in duplicates using CFX-96 real-time PCR device (Bio-rad). Mouse GAPDH (QT01658692) was used as housekeeping gene for all reactions.

Unc5B function-blocking antibody generation. We performed a Phage-Fab (antigen-binding fragment) selection using a naïve Fab library (lib $\mathrm{F}^{60}$ ) on a recombinant rat Unc5B-ECD Fc fusion protein (R\&D systems) previously immobilized directly to Nunc Maxisorp plates starting at $5 \mathrm{ug} / \mathrm{ml}$ and dropping by $1 \mathrm{ug} / \mathrm{ml}$ per round. Phage titers from both input and output were monitored daily to ensure daily inputs were prepared correctly (minimum input of $>1 \mathrm{x} 10 \mathrm{E} 10$ ) and output titers were at least 100-fold below input titers (day 1 output $\sim 1 \mathrm{x} 10 \mathrm{E} 3$, day 2 output $\sim 1 \mathrm{x}$ 10E4, day 3 output $\sim 1 \mathrm{x}$ 10E5, days 4 and 5 output $\sim 1 \mathrm{x}$ 10E7). Phage particles fused with Fabs were incubated with an unrelated protein (e.g., streptavidin) immobilized on a solid surface and allowed to bind in a step termed counterselection to remove unwanted phage-Fabs prior to incubation against target. After washing away unbound phage-Fab, phages were eluted from the target and amplified overnight for subsequent rounds. After 5 rounds of this process individual clones from rounds 3-5 were grown in 96-well format and tested by ELISA for their ability to bind antigen specifically. We selected several unique and different positive Fab over 5 rounds of selection, which were subcloned before antibody production (Proteogenix, Schiltigheim, France).

Surface plasmon resonance. Binding of anti-Unc5B antibodies to Human or Rat Unc5B was performed using a Biacore ${ }^{\mathrm{TM}} 8 \mathrm{~K}$ (Proteogenix, Schiltigheim, France). Human or Rat Unc5B-ECD-Fc (R\&D Systems) were immobilized on a CM5 sensor chip. Each antibody was diluted to gradient concentrations $(50 \mathrm{nM}, 25 \mathrm{nM}$, $12.5 \mathrm{nM}, 6.25 \mathrm{nM}, 3.125 \mathrm{nM}$ ) and flow through CM5 chip. The kinetic parameter was calculated using Bia-evaluation analysis software.

Intravenous injection of antibodies, fluorescent tracer and nanobodies. CTRL IgG, CTRL anti-Unc5B-1, anti-Unc5B-2 and -3 were injected intravenously into the lateral tail-vein of 8 weeks old adult mice at a concentration of $10 \mathrm{mg}$ of antibodies $/ \mathrm{kg}$ of mice and left to circulate from $1 \mathrm{~h}$ to $24 \mathrm{~h}$ depending on the experiment. All fluorescent tracers were injected intravenously into the lateral tail vein of 8 weeks old adult mice and left to circulate for $30 \mathrm{~min}$. Lysine-fixable Cadaverine conjugated to Alexa Fluor-555 (Invitrogen) was injected at a concentration of $100 \mathrm{ug}$ Cadaverine/20 g of mice. Lysine-fixable 10,40 or $70 \mathrm{kDa}$ dextran conjugated to tetramethylrhodamine (Invitrogen) were injected at a concentration of $250 \mathrm{ug}$ dextran/20 g of mice. Nanobodies (Alexa Fluor-488 coupled anti-mouse nanobodies, Abnova) were injected at a concentration of 60 ug nanobodies $/ 20 \mathrm{~g}$ of mice. For postnatal experiments, cadaverine was injected intraperitoneally into $\mathrm{P} 5$ neonates at a concentration of $250 \mathrm{ug}$ cadaverine $/ 20 \mathrm{~g}$ of pups and left to circulate for $2 \mathrm{~h}$.

Fluorescent tracer and nanobody quantification. To assess tracer and nanobody leak, animals were perfused in the left ventricle with PBS. Brains (and other organs) were then collected, and their weight measured. Next, brains (and other organs) were incubated in formamide (Sigma-Aldrich, F7503) at $56^{\circ} \mathrm{C}$ for $48 \mathrm{~h}$. Dye fluorescence was then measured using a spectrophotometer at the appropriate emission and excitation wavelength. Dye extravasation from Unc5B $B^{f / f}$, Unc5BiECko, WT treated with CTRL anti-Unc5B-1 and anti-Unc5B-2 were performed at the Yale Cardiovascular Research Center (New Haven, CT, USA) on a BioTek synergy2 spectrophotometer. Dye extravasation from WT treated with CTRL IgG2b and anti-Unc5B-3 were performed at the Paris Cardiovascular Research Center (Paris, France) on a Flexstation3 spectrophotometer. All results were normalized to the corresponding brain weight and reported to a standard made of known concentrations of dye diluted in formamide. Results are shown as $\mathrm{ng}$ of dye per $\mathrm{mg}$ of corresponding organ or tissue.

BDNF extravasation quantification. To assess BDNF leak, $1 \mathrm{~h}$ after antibody injection, $50 \mu \mathrm{g}$ of Human BDNF diluted in saline was injected intravenously into the lateral tail vein in adult mice and left to circulate for $30 \mathrm{~min}$. After sampling whole blood in EDTA-coated tubes, animals were perfused in the left ventricle with saline. Brains were then collected, and their weight measured. Blood was centrifuged at $1000 \times g$ for $15 \mathrm{~min}$ at $4{ }^{\circ} \mathrm{C}$, and plasma was then stored at $-20^{\circ} \mathrm{C}$ until use. Dissected brains were frozen in liquid nitrogen. They were lysed in RIPA buffer (Thermo Fisher) supplemented with protease and phosphatase inhibitor cocktails (Roche, 11836170001 and 4906845001) with increasing needle gauges and sonicated ( 3 times of $3 \mathrm{~min}$ each). All protein lysates were then centrifuged for $15 \mathrm{~min}$ at $14,000 \times g$ at $4{ }^{\circ} \mathrm{C}$ and supernatants were isolated.

BDNF concentration in the plasma and brain lysates was quantified using a DuoSet BDNF ELISA Kit (R\&D Systems, DY248) according to manufacturer instructions. Results were normalized to the corresponding brain weight. Results are shown as ng of nanobodies per $\mathrm{mg}$ of brain tissue.

Two-photon live imaging. Craniotomy was performed by drilling a 5-mm circle between lambdoid, sagittal, and coronal sutures of the skull on ketamine/xylazine anesthetized WT mice. After skull removal, the cortex was sealed with a glass coverslip cemented on top of the mouse skull. Live imaging was done 2 weeks later For multiphoton excitation of endogenous fluorophores and injected dyes, we used a Leica SP8 DIVE in vivo imaging system equipped with 4tune spectral external hybrid detectors and an InSightX3 laser (SpectraPhysics). The microscope was equipped with in house designed mouse holding platform for intravital imaging (stereotactic frame, Narishige; gas anesthesia and body temperature monitoring/ control, Minerve). Mice were injected intravenously with $10 \mathrm{mg} / \mathrm{kg}$ of CTRL IgG or anti-Unc5B-3 for $1 \mathrm{~h}$ followed by i.v injection of $10 \mathrm{kDa}$ dextran for $1 \mathrm{~h}$ and image acquisition. 
Confocal microscopy and image analysis. Confocal images were acquired on a laser scanning fluorescence microscope (Leica SP8 and Zeiss LSM800) using the appropriate software (LASX or ZEN system). 10X, 20X and 63X oil immersion objectives were used for acquisition using selective laser excitation $(405,488,547$, or $647 \mathrm{~nm}$ ). Vascular density was quantified with the software Angiotool by quantifying the vascular surface area normalized to the total surface area. Quantification of pixel intensity was performed using the software ImageJ. VE-cadherin coverage analysis was performed on ImageJ by quantifying the VE-cadherin + area normalized to the podocalyxin + area. Vessel diameter was calculated on Leica LASX software.

Statistical analysis. All in vivo experiments were done on littermates with similar body weight per condition. Statistical analysis was performed using GraphPad Prism 8 software. Two-sided Mann-Whitney U test was performed for statistical analysis of two groups. ANOVA followed by Bonferroni's multiple comparisons test was performed for statistical analysis between 3 or more groups. Mantel-cox test was performed for survival curve statistical analysis.

Reporting summary. Further information on research design is available in the Nature Research Reporting Summary linked to this article.

\section{Data availability}

All data generated are included in this article (main or supplementary information files) and are provided in the Source Data file. Additional information can be obtained from the corresponding author. Source data are provided with this paper.

Received: 14 May 2021; Accepted: 18 January 2022;

Published online: 04 March 2022

\section{References}

1. Zhao, Z., Nelson, A. R., Betsholtz, C. \& Zlokovic, B. V. Establishment and dysfunction of the blood-brain barrier. Cell 163, 1064-1078 (2015).

2. Obermeier, B., Daneman, R. \& Ransohoff, R. M. Development, maintenance and disruption of the blood-brain barrier. Nat. Med. 19, 1584-1596 (2013).

3. Daneman, R. \& Prat, A. The blood-brain barrier. Cold Spring Harb. Perspect. Biol. 7, a020412 (2015).

4. Nitta, T. et al. Size-selective loosening of the blood-brain barrier in claudin-5deficient mice. J. Cell Biol. 161, 653-660 (2003).

5. Luissint, A. C., Artus, C., Glacial, F., Ganeshamoorthy, K. \& Couraud, P. O. Tight junctions at the blood brain barrier: physiological architecture and disease-associated dysregulation. Fluids Barriers CNS 9, 23 (2012).

6. Andreone, B. J. et al. Blood-brain barrier permeability is regulated by lipid transport-dependent suppression of caveolae-mediated transcytosis. Neuron 94, 581-594 e585 (2017).

7. Ben-Zvi, A. et al. Mfsd2a is critical for the formation and function of the blood-brain barrier. Nature 509, 507-511 (2014).

8. Simpson, I. A., Carruthers, A. \& Vannucci, S. J. Supply and demand in cerebral energy metabolism: the role of nutrient transporters. J. Cereb. Blood Flow. Metab. 27, 1766-1791 (2007).

9. Loscher, W. \& Potschka, H. Blood-brain barrier active efflux transporters: ATP-binding cassette gene family. NeuroRx 2, 86-98 (2005).

10. De Vivo, D. C. et al. Defective glucose transport across the blood-brain barrier as a cause of persistent hypoglycorrhachia, seizures, and developmental delay. N. Engl. J. Med. 325, 703-709 (1991).

11. Armulik, A. et al. Pericytes regulate the blood-brain barrier. Nature 468, 557-561 (2010).

12. Daneman, R., Zhou, L., Kebede, A. A. \& Barres, B. A. Pericytes are required for blood-brain barrier integrity during embryogenesis. Nature 468, 562-566 (2010).

13. Zonta, M. et al. Neuron-to-astrocyte signaling is central to the dynamic control of brain microcirculation. Nat. Neurosci. 6, 43-50 (2003).

14. Pulido, R. S. et al. Neuronal activity regulates blood-brain barrier efflux transport through endothelial circadian genes. Neuron 108, 937-952 e937 (2020).

15. Clevers, H. Wnt/beta-catenin signaling in development and disease. Cell 127, 469-480 (2006).

16. Zhou, Y. \& Nathans, J. Gpr124 controls CNS angiogenesis and blood-brain barrier integrity by promoting ligand-specific canonical wnt signaling. Dev. Cell 31, 248-256 (2014).

17. Eubelen, M. et al. A molecular mechanism for Wnt ligand-specific signaling. Science 361 https://doi.org/10.1126/science.aat1178 (2018).

18. Cho, C., Smallwood, P. M. \& Nathans, J. Reck and Gpr124 Are Essential Receptor Cofactors for Wnt7a/Wnt7b-Specific Signaling in Mammalian CNS Angiogenesis and Blood-Brain Barrier Regulation. Neuron 95, 1221-1225 (2017).
19. Chang, J. et al. Gpr124 is essential for blood-brain barrier integrity in central nervous system disease. Nat. Med 23, 450-460 (2017)

20. Wang, Y. et al. Norrin/Frizzled4 signaling in retinal vascular development and blood brain barrier plasticity. Cell 151, 1332-1344 (2012).

21. Wang, Y. et al. Interplay of the Norrin and Wnt7a/Wnt7b signaling systems in blood-brain barrier and blood-retina barrier development and maintenance. Proc. Natl Acad. Sci. USA 115, E11827-E11836 (2018).

22. Liebner, $\mathrm{S}$. et al. Wnt/beta-catenin signaling controls development of the blood-brain barrier. J. Cell Biol. 183, 409-417 (2008).

23. Daneman, R. et al. Wnt/beta-catenin signaling is required for CNS, but not non-CNS, angiogenesis. Proc. Natl Acad. Sci. USA 106, 641-646 (2009).

24. Zhou, Y. et al. Canonical WNT signaling components in vascular development and barrier formation. J. Clin. Invest 124, 3825-3846 (2014).

25. Leung-Hagesteijn, C. et al. UNC-5, a transmembrane protein with immunoglobulin and thrombospondin type 1 domains, guides cell and pioneer axon migrations in C. elegans. Cell 71, 289-299 (1992).

26. Leonardo, E. D. et al. Vertebrate homologues of C. elegans UNC-5 are candidate netrin receptors. Nature 386, 833-838 (1997).

27. $\mathrm{Lu}, \mathrm{X}$. et al. The netrin receptor UNC5B mediates guidance events controlling morphogenesis of the vascular system. Nature 432, 179-186 (2004).

28. Larrivee, B. et al. Activation of the UNC5B receptor by Netrin-1 inhibits sprouting angiogenesis. Genes Dev. 21, 2433-2447 (2007).

29. Koch, A. W. et al. Robo4 maintains vessel integrity and inhibits angiogenesis by interacting with UNC5B. Dev. Cell 20, 33-46 (2011).

30. Zhang, F. et al. The Robo4 cytoplasmic domain is dispensable for vascular permeability and neovascularization. Nat. Commun. 7, 13517 (2016).

31. Tai-Nagara, I. et al. Placental labyrinth formation in mice requires endothelial FLRT2/UNC5B signaling. Development 144, 2392-2401 (2017).

32. Yamagishi, S. et al. FLRT2 and FLRT3 act as repulsive guidance cues for Unc5-positive neurons. EMBO J. 30, 2920-2933 (2011).

33. Wang, R. et al. Autoinhibition of UNC5b revealed by the cytoplasmic domain structure of the receptor. Mol. Cell 33, 692-703 (2009).

34. Llambi, F., Causeret, F., Bloch-Gallego, E. \& Mehlen, P. Netrin-1 acts as a survival factor via its receptors UNC5H and DCC. EMBO J. 20, 2715-2722 (2001).

35. Wang, Y. et al. Ephrin-B2 controls VEGF-induced angiogenesis and lymphangiogenesis. Nature 465, 483-486 (2010).

36. Horvat, R., Hovorka, A., Dekan, G., Poczewski, H. \& Kerjaschki, D. Endothelial cell membranes contain podocalyxin-the major sialoprotein of visceral glomerular epithelial cells. J. Cell Biol. 102, 484-491 (1986).

37. Cuervo, H. et al. PDGFRbeta-P2A-CreER(T2) mice: a genetic tool to target pericytes in. Angiogenesis. Angiogenesis 20, 655-662 (2017).

38. Bauer, H. \& Traweger, A. Tight junctions of the blood-brain barrier - a molecular gatekeeper. CNS Neurol. Disord. Drug Targets 15, 1016-1029 (2016).

39. Ferrer-Vaquer, A. et al. A sensitive and bright single-cell resolution live imaging reporter of Wnt/ss-catenin signaling in the mouse. BMC Dev. Biol. 10, 121 (2010).

40. Birdsey, G. M. et al. Transcription factor Erg regulates angiogenesis and endothelial apoptosis through VE-cadherin. Blood 111, 3498-3506 (2008).

41. Niehrs, C. \& Shen, J. Regulation of Lrp6 phosphorylation. Cell Mol. Life Sci. 67, 2551-2562 (2010).

42. Mao, B. et al. LDL-receptor-related protein 6 is a receptor for Dickkopf proteins. Nature 411, 321-325 (2001)

43. Brault, V. et al. Inactivation of the beta-catenin gene by Wnt1-Cre-mediated deletion results in dramatic brain malformation and failure of craniofacial development. Development 128, 1253-1264 (2001)

44. Harada, N. et al. Intestinal polyposis in mice with a dominant stable mutation of the beta-catenin gene. EMBO J. 18, 5931-5942 (1999).

45. Wang, Y. et al. Beta-catenin signaling regulates barrier-specific gene expression in circumventricular organ and ocular vasculatures. Elife $\mathbf{8}$ https:// doi.org/10.7554/eLife.43257 (2019).

46. Knowland, D. et al. Stepwise recruitment of transcellular and paracellular pathways underlies blood-brain barrier breakdown in stroke. Neuron $\mathbf{8 2}$, 603-617 (2014).

47. Taddei, A. et al. Endothelial adherens junctions control tight junctions by VEcadherin-mediated upregulation of claudin-5. Nat. Cell Biol. 10, 923-934 (2008).

48. Li, X. et al. VEGFR2 pY949 signalling regulates adherens junction integrity and metastatic spread. Nat. Commun. 7, 11017 (2016)

49. Vanlandewijck, M. et al. A molecular atlas of cell types and zonation in the brain vasculature. Nature 554, 475-480 (2018).

50. Brunet, I. et al. Netrin-1 controls sympathetic arterial innervation. J. Clin. Invest 124, 3230-3240 (2014).

51. Huyghe, A. et al. Netrin-1 promotes naive pluripotency through Neol and Unc5b co-regulation of Wnt and MAPK signalling. Nat. Cell Biol. 22, 389-400 (2020). 
52. Podjaski, C. et al. Netrin 1 regulates blood-brain barrier function and neuroinflammation. Brain 138, 1598-1612 (2015).

53. Yao, L. L. et al. Astrocytic neogenin/netrin-1 pathway promotes blood vessel homeostasis and function in mouse cortex. J. Clin. Invest. 130, 6490-6509 (2020).

54. Nedeva, I. et al. Relationship between circulating netrin-1 levels, obesity, prediabetes and newly diagnosed type 2 diabetes. Arch Physiol. Biochem., 1-6 https://doi.org/10.1080/13813455.2020.1780453 (2020).

55. Idbaih, A. et al. Safety and feasibility of repeated and transient blood-brain barrier disruption by pulsed ultrasound in patients with recurrent glioblastoma. Clin. Cancer Res. 25, 3793-3801 (2019).

56. Carpentier, A. et al. Clinical trial of blood-brain barrier disruption by pulsed ultrasound. Sci. Transl. Med 8, 343re342 (2016).

57. Sheikov, N., McDannold, N., Vykhodtseva, N., Jolesz, F. \& Hynynen, K. Cellular mechanisms of the blood-brain barrier opening induced by ultrasound in presence of microbubbles. Ultrasound Med. Biol. 30, 979-989 (2004).

58. Aryal, M., Arvanitis, C. D., Alexander, P. M. \& McDannold, N. Ultrasoundmediated blood-brain barrier disruption for targeted drug delivery in the central nervous system. Adv. Drug Deliv. Rev. 72, 94-109 (2014).

59. Munji, R. N. et al. Profiling the mouse brain endothelial transcriptome in health and disease models reveals a core blood-brain barrier dysfunction module. Nat. Neurosci. 22, 1892-1902 (2019).

60. Persson, $\mathrm{H}$. et al. CDR-H3 diversity is not required for antigen recognition by synthetic antibodies. J. Mol. Biol. 425, 803-811 (2013).

\section{Acknowledgements}

This project has received funding from the NIH (1R01HL149343-01 to A.E., 5R01NS35900 to S.L.A.), the Leducq Foundation (TNE ATTRACT, AE, LCW) and the European Research Council (ERC) (grant agreement No. 834161 to A.E.). K.B. was supported by a fellowship from the AHA (18POST34070109). We thank Max Thomas for technical assistance. S.L.A. is an investigator of the Howard Hughes Medical Institute.

\section{Author contributions}

K.B., L.H.G., J.F., L.P.F., M.P., Y.X., L.J. and N.M. performed experiment. K.B. and A.E. designed experiments and analyzed data. A.E. and K.B. wrote the manuscript. D.K, B.N., D.A, L.C.W. and S.L.A., generated and provided essential reagents/mice.

\section{Competing interests}

A.E., K.B., L.G. and L.P-F. are inventors on patent applications that cover the generation of Unc5B blocking antibodies, and their application. The remaining authors declare no competing interests.

\section{Additional information}

Supplementary information The online version contains supplementary material available at https://doi.org/10.1038/s41467-022-28785-9.

Correspondence and requests for materials should be addressed to Anne Eichmann.

Peer review information Nature Communications thanks Elisabetta Dejana and the other anonymous reviewers for their contribution to the peer review of this work. Peer reviewer reports are available.

Reprints and permission information is available at http://www.nature.com/reprints

Publisher's note Springer Nature remains neutral with regard to jurisdictional claims in published maps and institutional affiliations.

(c) (i) Open Access This article is licensed under a Creative Commons Attribution 4.0 International License, which permits use, sharing, adaptation, distribution and reproduction in any medium or format, as long as you give appropriate credit to the original author(s) and the source, provide a link to the Creative Commons license, and indicate if changes were made. The images or other third party material in this article are included in the article's Creative Commons license, unless indicated otherwise in a credit line to the material. If material is not included in the article's Creative Commons license and your intended use is not permitted by statutory regulation or exceeds the permitted use, you will need to obtain permission directly from the copyright holder. To view a copy of this license, visit http://creativecommons.org/ licenses/by/4.0/.

(C) The Author(s) 2022 